UNITED STATES DEPARTMENT OF THE INTERIOR

GEOLOGICAL SURVEY

\title{
Geochemical Analyses of Rock, Stream-Sediment, and Heavy-Mineral Concentrate Samples Collected from the Spanish Peaks Wilderness Study Area, Huerfano and Las Animas Counties, Colorado
}

\section{By}

Karin E. Budding and Viki A. Lawrence

Open-File Report 83-391

1983

This report is preliminary and has not been reviewed for conformity with U.S. Geological Survey editorial standards. 


\section{STUDIES RELATED TO WILDERNESS}

The Wilderness Act (Public Law 88-577, September 3, 1964) and related acts require the U.S. Geological Survey and the U.S. Bureau of Mines to survey certain areas on Federal lands to determine their mineral resource potential. Results must be made available to the public and be submitted to the President and the Congress. This report presents the results of a geochemical survey of the Spanish Peaks Wilderness Study Area in the San Isabel National Forest, Huerfano and Las Animas Counties, Colorado. The Spanish Peaks Wilderness Study Area was established by Public Law 96-560, December 22, 1980.

\section{INTRODUCTION}

The Spanish Peaks Wilderness Study Area covers approximately 19,570 acres $\left(7,920\right.$ hectometers $\left.{ }^{2}\right)$ of the San Isabel National Forest in south-central Colorado (fig. 1). The area lies in the westernmost part of the Great Plains, bordering the eastern foothills of the Sangre de Cristo Mountains. Elevations range from $13,626 \mathrm{ft}(4,153 \mathrm{~m})$ on the summit of West Spanish Peak to about $8,400 \mathrm{ft}(2,560 \mathrm{~m})$ in the western portion near Cuchara. The eastern half of the wilderness is characterized by rugged terrain; the land and drainages slope radially away from East and West Spanish Peaks. The principal drainages are Wahatoya and Trujillo Creeks. The physiography in the western half of the area is less severe; the major drainages are the north-trending Chaparral and Echo Creeks. North, Middle, and South White Peaks (elevation 10,446 ft; $3,184 \mathrm{~m}$ ) are located near the western boundary of the study area.

Colorado Highway 12 and the Cucharas River parallel the wilderness on the west. Forest Service Route 415 and the Apishapa River run along the southern margin. Secondary roads are sparsely located near the boundary of the study area.

This report tabulates the geochemical data from the rock, stream sediment, and heavy-mineral (panned) concentrate samples collected to assist in the assessment of the mineral resource potential of the Spanish Peaks Wilderness Study Area, Colorado. In a separate series of Miscellaneous Field Studies Maps, Budding and Lawrence discuss geochemical results and identify the sample localities referenced in this report (1983a), show a geologic map (1983b), and Budding and Kluender (1983) evaluate the mineral resource potential of this study area. 


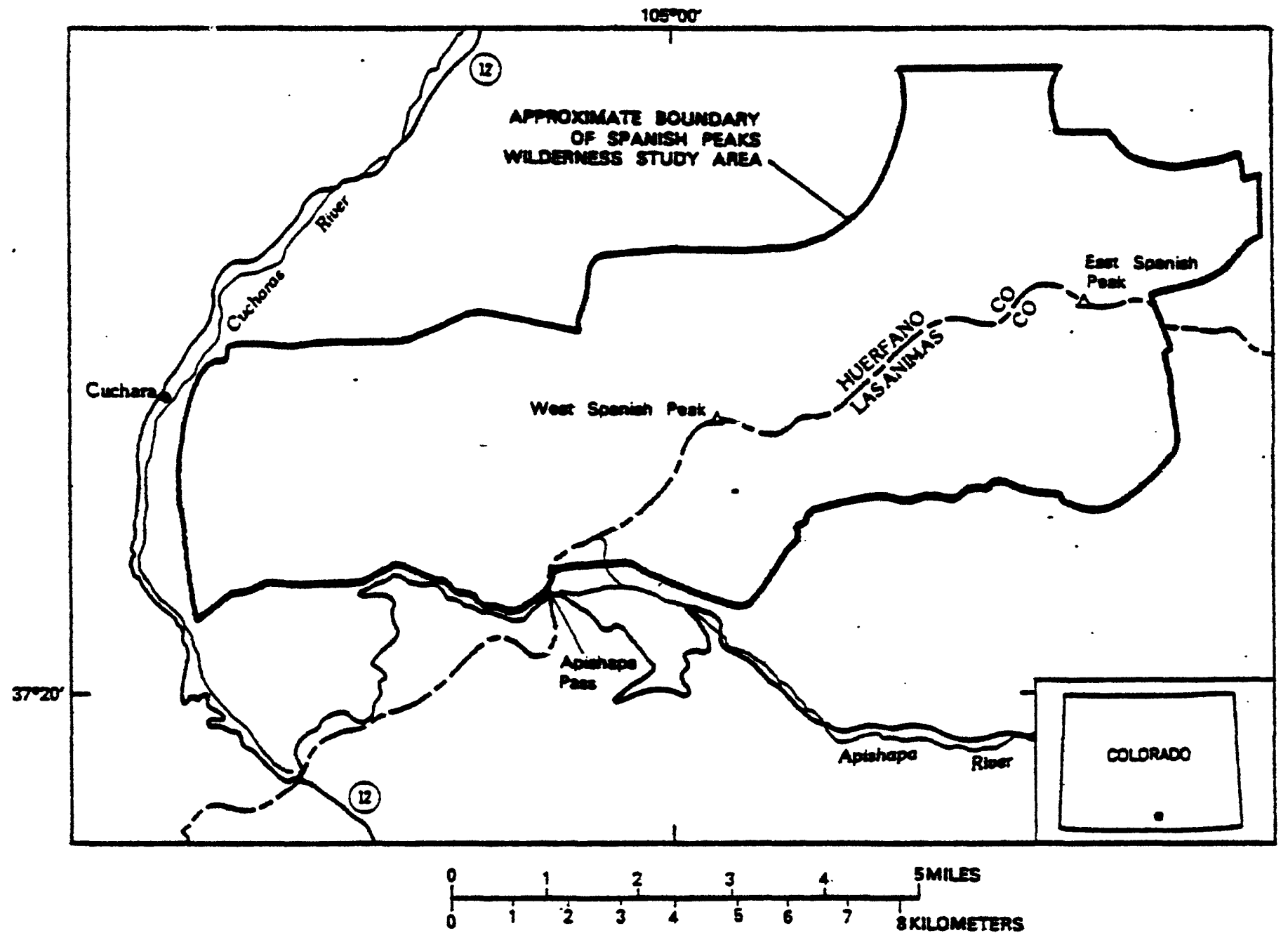

Figure 1.-Index map showing location of the Spanish Peaks Wilderness Study Area. 
Table 1.--Lower limits of detection for semiquantitative emission spectrographic analyses of samples of rock, stream sediment, and heavy-mineral concentrate

[Limits calculated in percent for $\mathrm{Fe}, \mathrm{Mg}, \mathrm{Ca}, \mathrm{Ti}$, and in parts per million for a11 other elements]

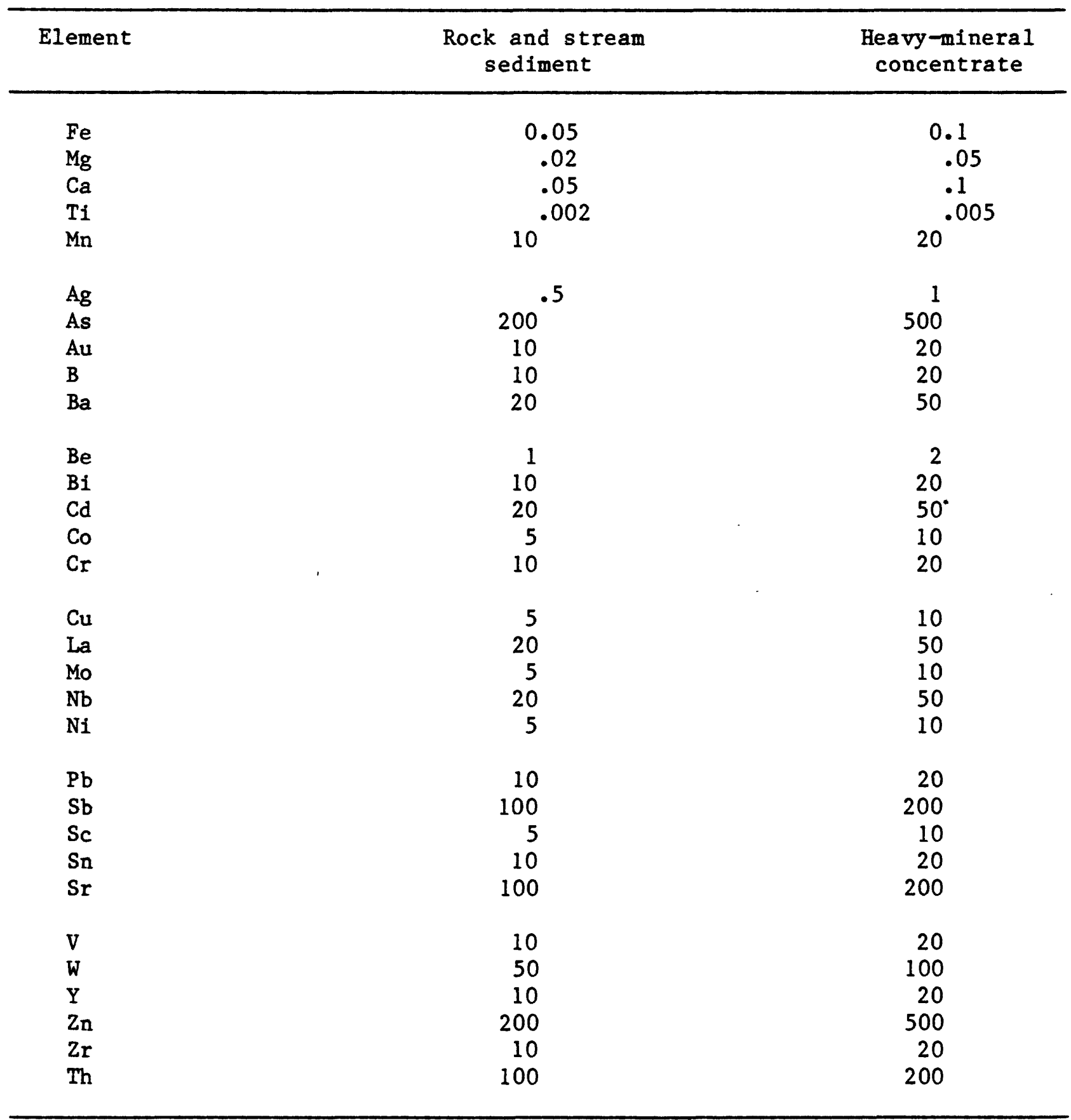


Table 2.--Data for rock samples

[Concentrations in percent for $\mathrm{Fe}, \mathrm{Mg}, \mathrm{Ca}, \mathrm{Ti}$, and in parts per million for all other elements; analyses where the column is headed by the letter s were by semiquantitative emission spectrometry; analyses where the column is headed by the letters aa were by atomic absorption; and $U$ analyses were by fluorimetry; $N$, not detected; leaders (-), not analyzed] 
竞

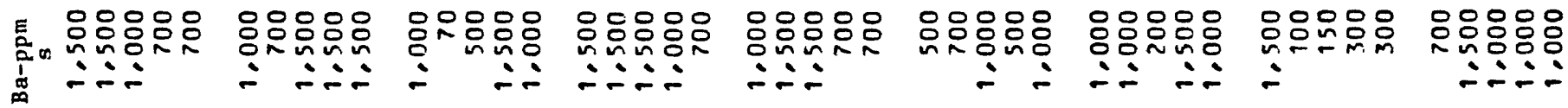

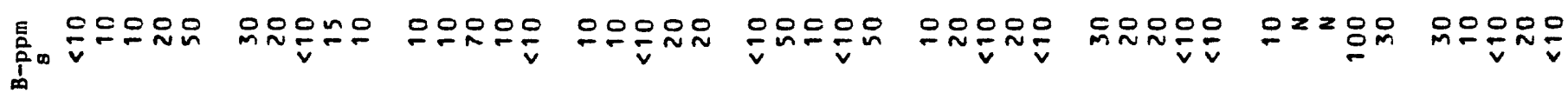

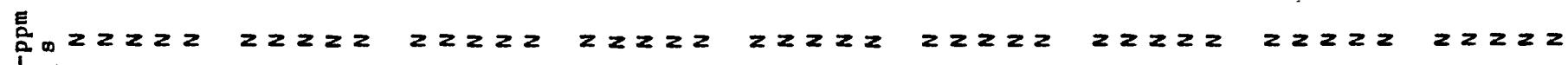
$\frac{1}{2}-1$

${ }_{\text {E. }}^{E} z z z z z z z z z$ z

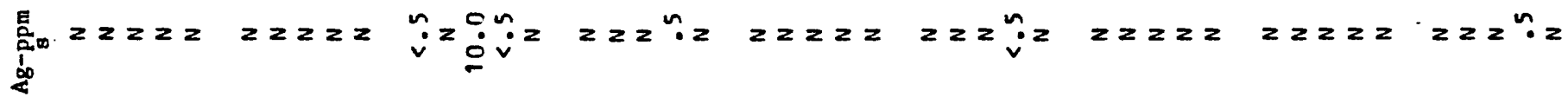

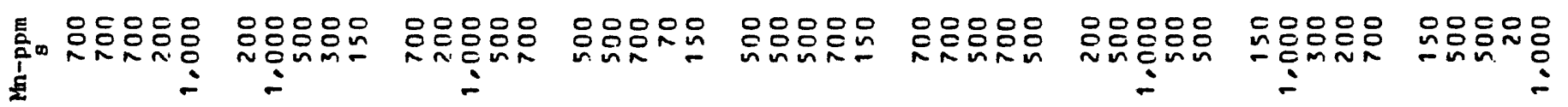

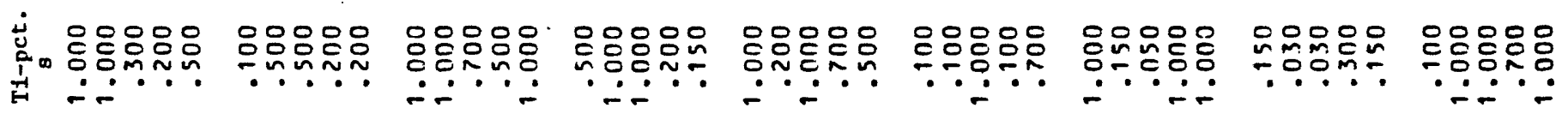

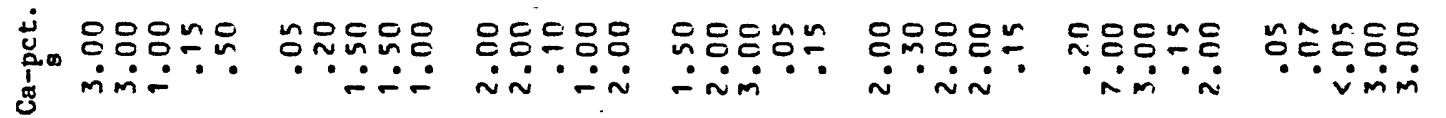

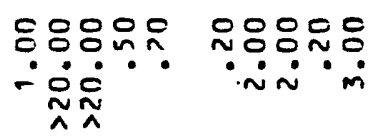

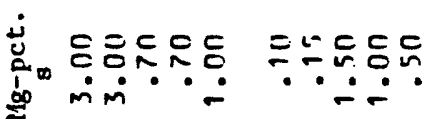
든등동등

อธ유 드으은 cecos eccoc

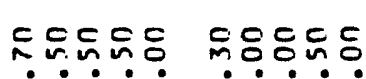

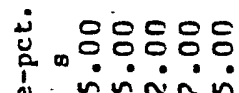
$\because \ddot{n} \dot{n}$

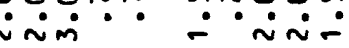
$\because$ กั 웅으응

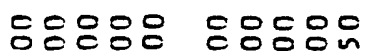
0000 जिтिंखि ำ.응 nivini-

든ㄷㅇㅇ응응

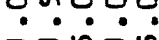

응등등융 nंn் 등웅응 $\therefore \therefore \dot{0}$

둥ㅇㅇ응 rinivin nnonn

ำกำ moñ z...

ngoo 으요

동영요요 든영 wํㅠㅁำ $\infty \infty \infty \infty \infty$ $\sim \tilde{n} \tilde{n} \sim$ in $\operatorname{mos}$ $\infty \propto \infty \infty 0$

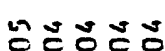
ธี่์ณn

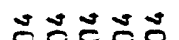
r. un $n$ a a a a c n m 0 n ๓n๊ュำ

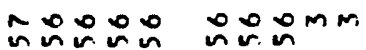
nnomm $\operatorname{mon} n$ 음는

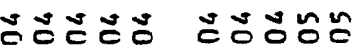

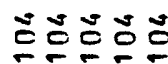

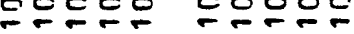

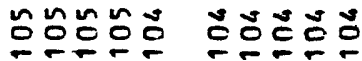
Oño0 o m nm onomn nnomo onnom nonom $\because n 000$

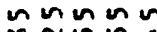
ณ

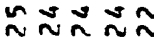
$\tilde{N} \tilde{\sim} \tilde{\sim}$ $\tilde{\sim} \sim \tilde{\sim} \sim$ 요묘 $\approx \approx \approx \approx \approx$ ニニ்̃ miñ $\sim \sim N$ $\approx \approx \approx m \bar{n}$ $\hat{M} \tilde{m} \tilde{M} \tilde{m}$ $\hat{M} \hat{N} \tilde{M} \hat{M}$

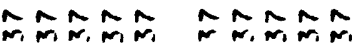

$\hat{m} \hat{m} \hat{m} \hat{m} \hat{m}$
$\hat{M} \hat{M} \hat{N}_{1} \hat{N}_{1} \hat{N}_{1} \hat{M} \tilde{M} \hat{M}_{1} \hat{N_{1}}$
N. N.MNAN $m \sim \tilde{\sim} \tilde{N}$ $\hat{m} \hat{m} \hat{m} \tilde{m}$

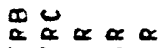

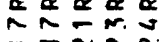

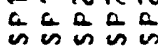

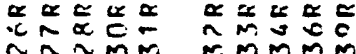
क $2=5<$

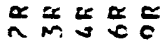
is is is is is
웅

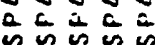

$\therefore$ nuㄷㅇ

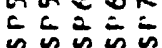

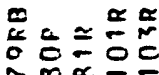
它

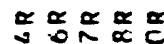
อ้ํํํํㅇํㅇ is

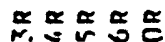

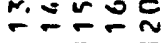
五: $=$

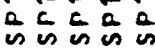


E

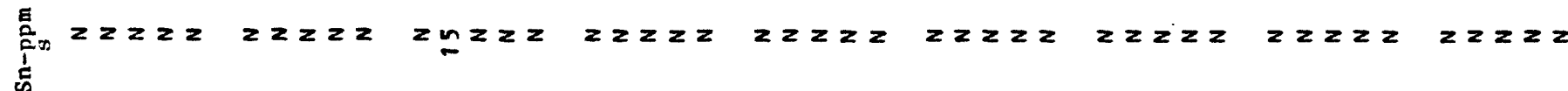
$-i$

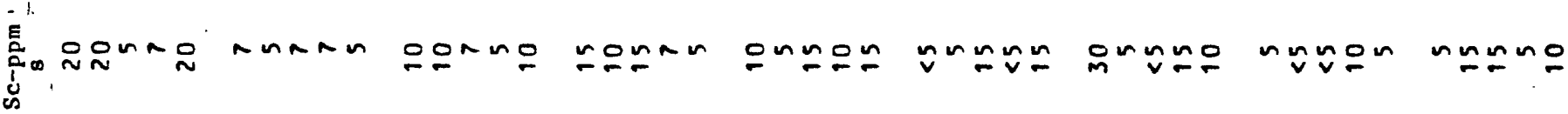

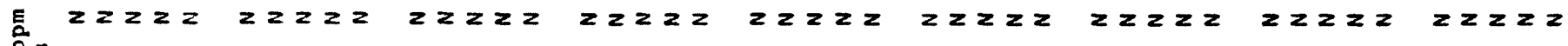
is.

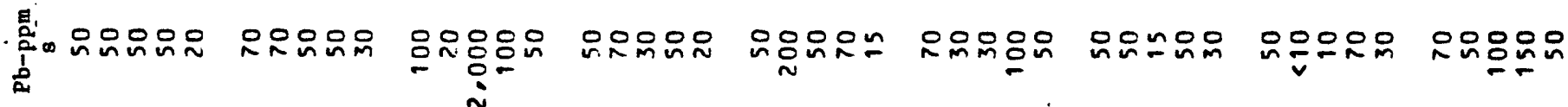
$\cdots$

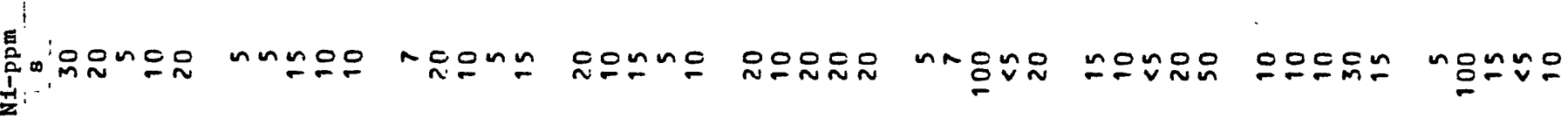

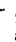
总

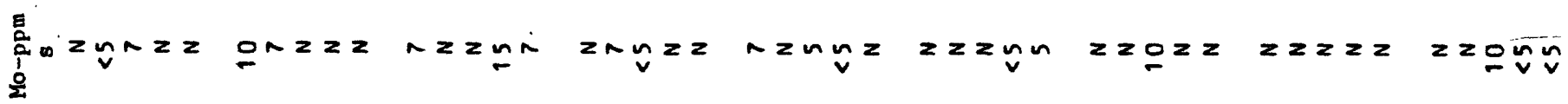

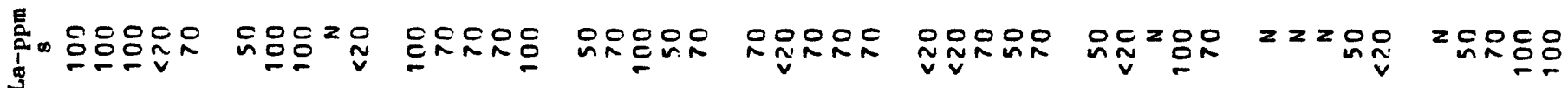
恶 $\cdots$

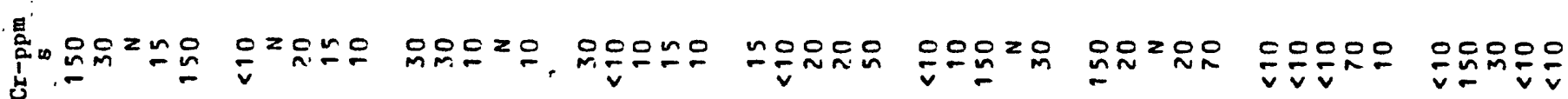

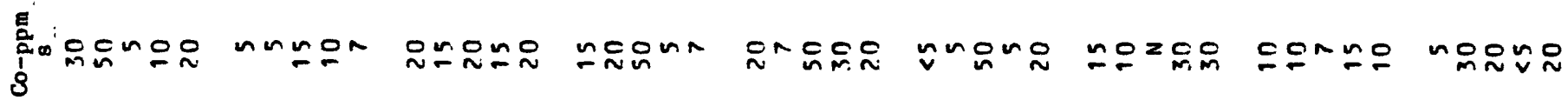

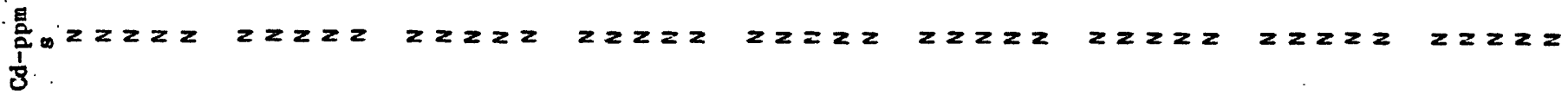

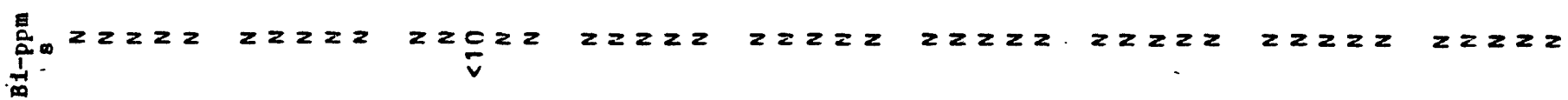




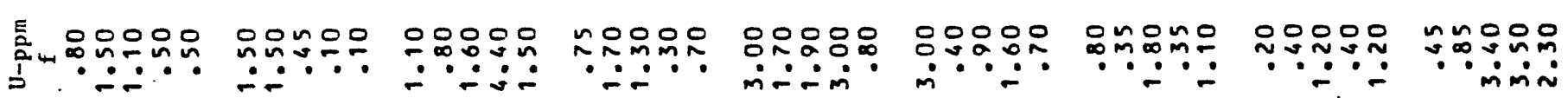

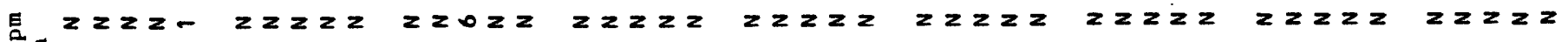
$\overbrace{}^{1}$

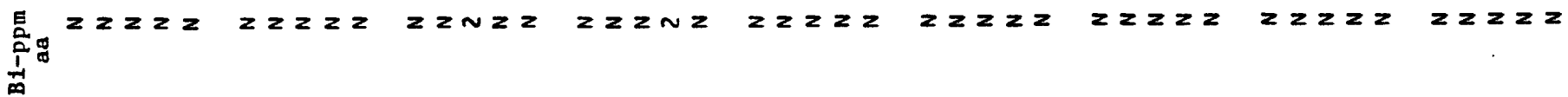

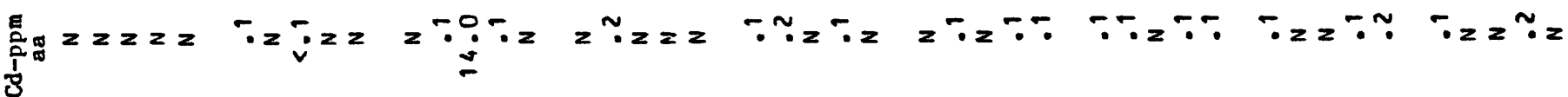

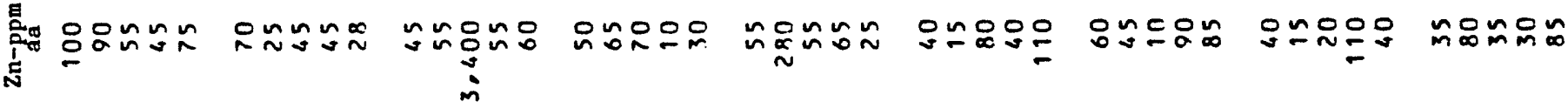
E

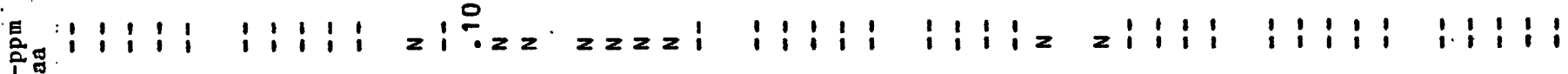
当.

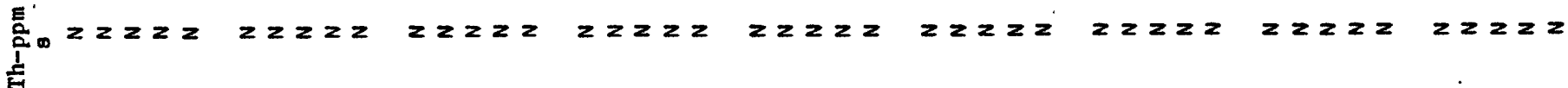

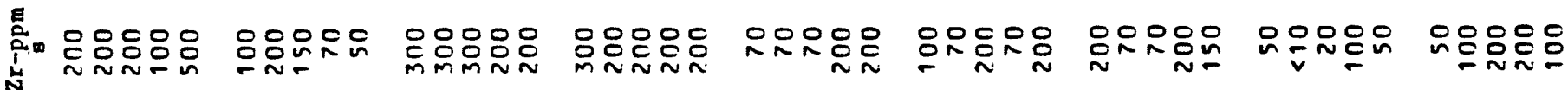

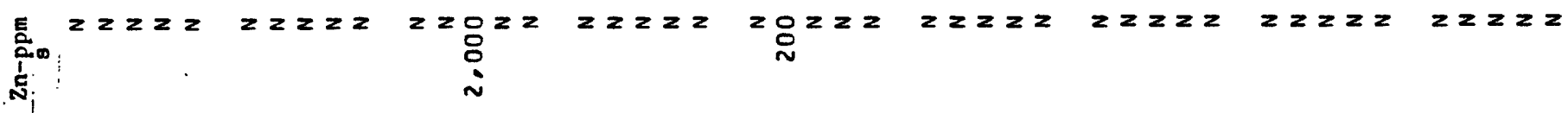

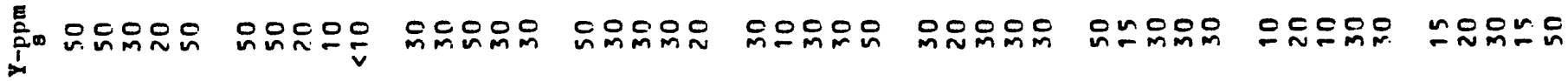

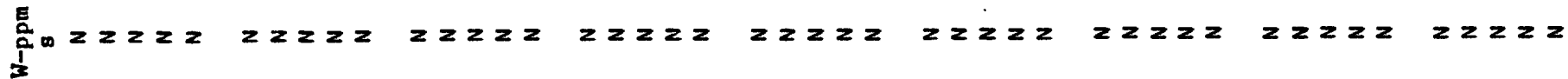
틀

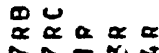

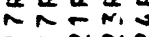
- - N N $\propto \alpha$

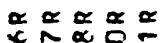
수수음

$\propto \approx \approx \approx$ กิm m.m ma $\stackrel{\sim}{\sim} \approx \approx$ ษงษ जेaे a is is is

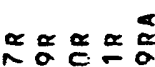
숭ㄷㅇ ññ 的的的的

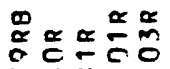
a o $x=$

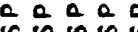

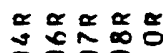
둥요 a ca a is is is is 원 ニー a a a a 的的的是 


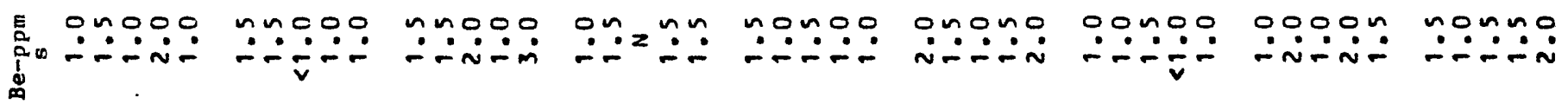

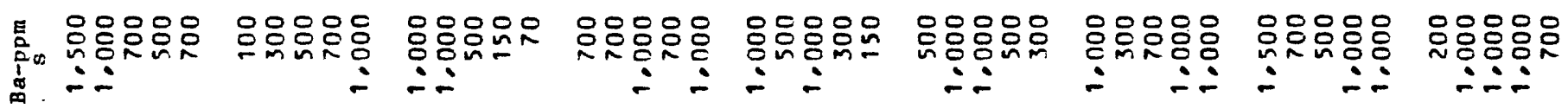

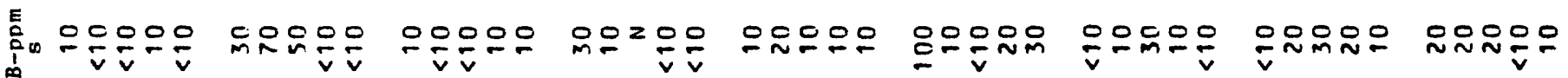

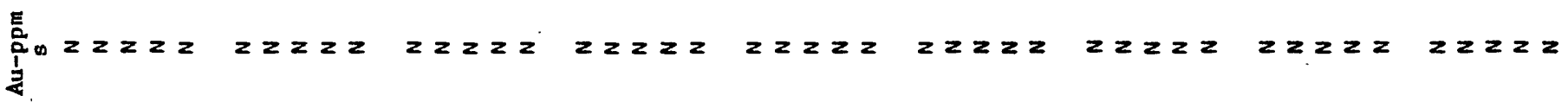

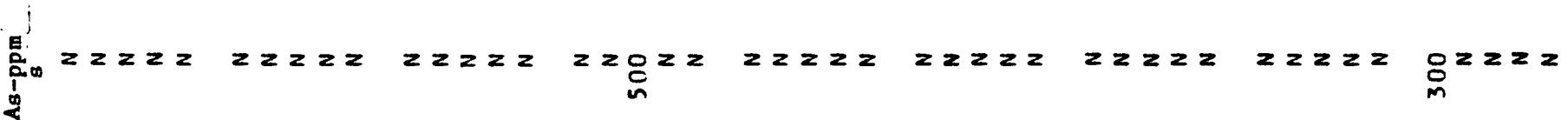

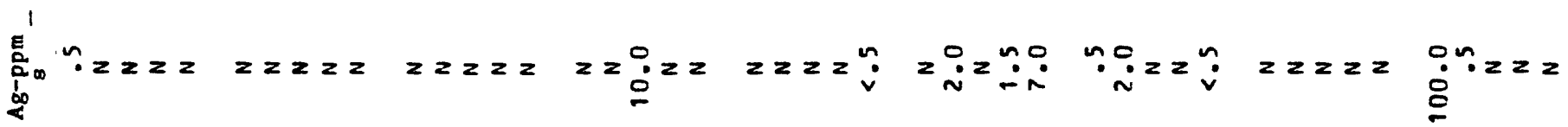
|

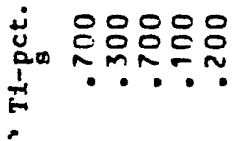

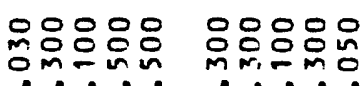

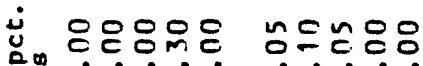

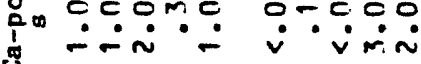

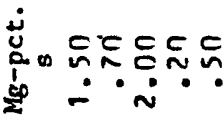
드ํํํํํํำ 등ㄷํㄷำ

옹응융ํำ

등등등ㅇㅇㅇ レே・ோ

유ㅇㅠㅠํำ

으읃응용응

8

옹든응ㅇㅇㅇ - $\dot{\sim} \dot{\sim}$.

읃ㅇㅇㅇㅇㅇㅇㅇㅇㅇ

8

응은응응

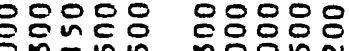
!.!.? mo:?

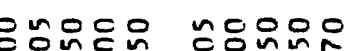
m.ñ

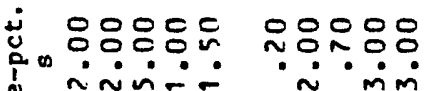
응ㅇㅇㅇㅇㅇ ․․․․

응응둥응

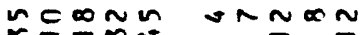

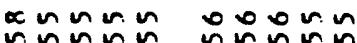

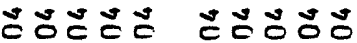
믄 듀음요

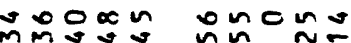
NMmNM mms

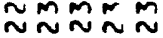
$\hat{M} \tilde{m} \tilde{m} \hat{m} \tilde{m}$ mãñ $\hat{M} \tilde{M} M \hat{M}$

느믄 $n n n n n$ num num ठัธ์ํํํำ

$\infty 0 \infty \infty \infty$ $n$ $\sim \infty \cdots \infty \infty$ เป็ัல 등ㅇㅇㅇㅇㅇ

$\approx \sim \approx-\infty$ $n \pi n \sim n$ ñññ

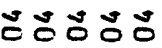

든 $\sim \sim \sim \sim \infty$ nnminn ธิธั0லั 드은

$n \infty m \sim n$ $00 \infty 0 \pi$ n NNMm $\approx \approx \approx \approx \tilde{\sim}$

$\approx \sim \approx \sim \approx$

บับำㅇำ $\hat{M} \hat{m} \hat{N} \hat{m} \tilde{m}$

$\hat{M} \tilde{m} \tilde{m} \tilde{m}$

$\hat{m} \tilde{m} \tilde{m} \hat{m} \tilde{m}$

$\hat{m} \hat{m} \hat{m} \hat{m}$
응ㅇㅇㅇㅇㅇ - $\dot{0} \dot{n}$

응응ㅇㅇㅇ 누의

응ㅇㅇㅇㅇㅇㅇㅇㅇㅇ -minio

응ㄷㅇㅇㅇㅇㅇㅇ inim

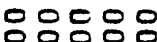
$\therefore .0 .0$ $\dot{m} \dot{m}-\dot{n}$ 문 緔

$\sim \sim \sim \sim \sim$ oon in $\infty \propto \infty v-$

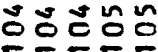

$=0$ mog $\approx \approx \approx \approx \sim ⿻ 上 丨$ $\hat{m} N \hat{m} N$ $\hat{m} \tilde{m} \hat{m} \hat{m}$

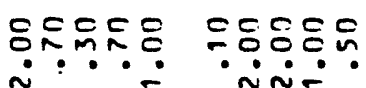

$\approx \approx \alpha \alpha$ a a a a ox 00 nocon N $\sim$ -

$\approx \infty 000$

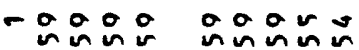

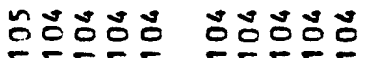

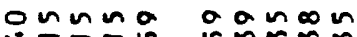
r.mmmn $\approx \sim \sim m m$

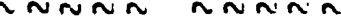

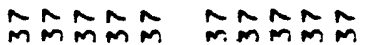

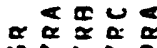
Áñ -mmm 的的它的 ñña

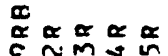

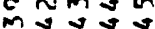

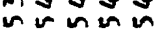
a c a c a

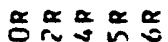
vinn ตัตร 矮的的的 $\alpha \approx \alpha \alpha$

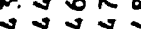

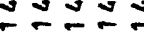
a a a

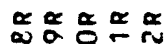

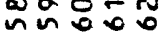
c. $-\overline{0}-\frac{1}{0}$

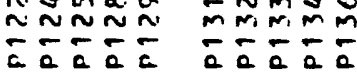
的的的的 


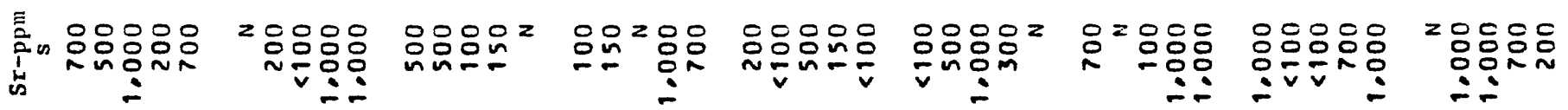

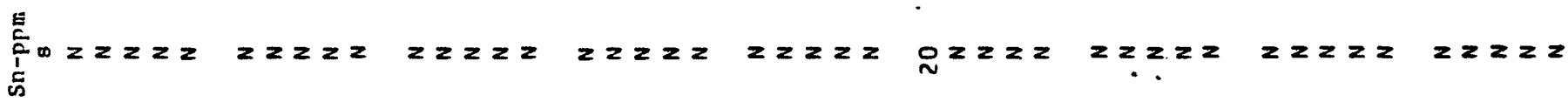
E⿱丷⿹弔㇒

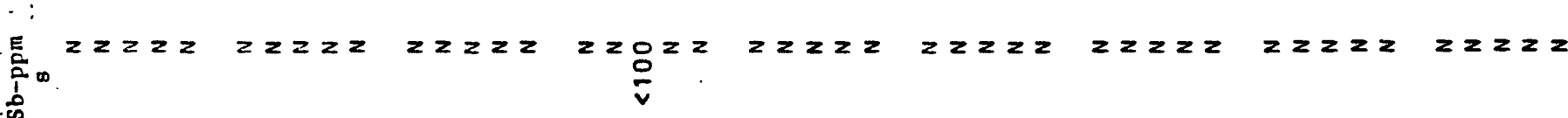

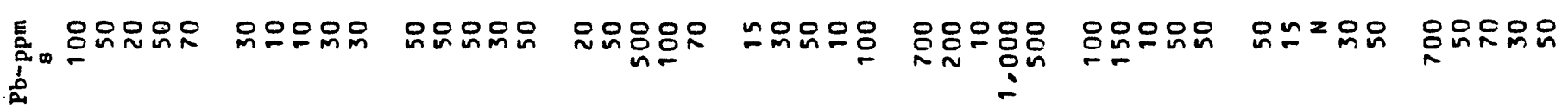

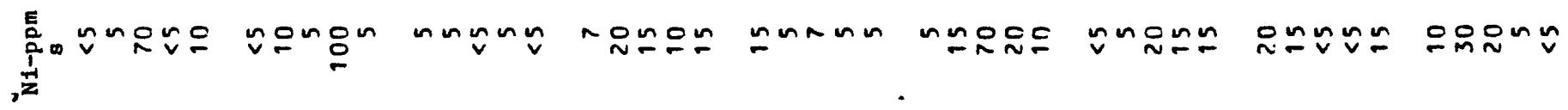

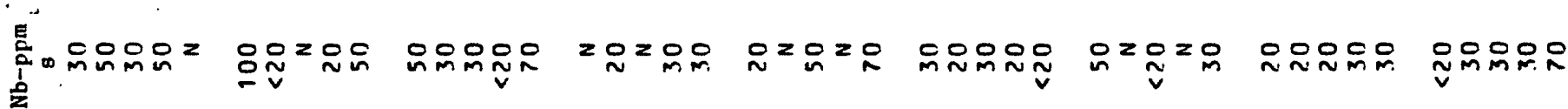

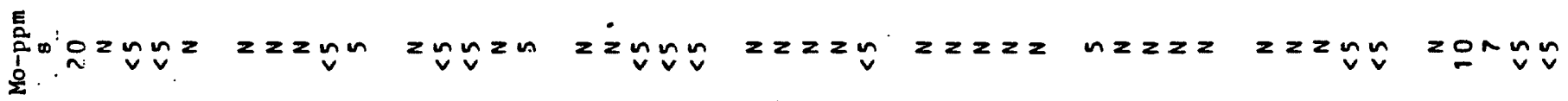
㶪

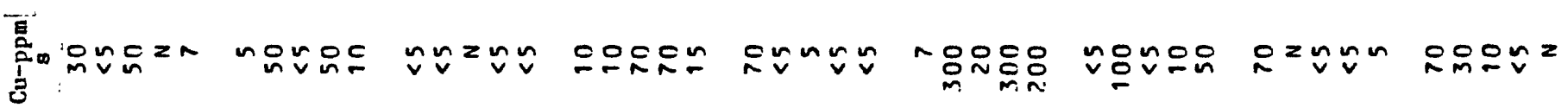
家

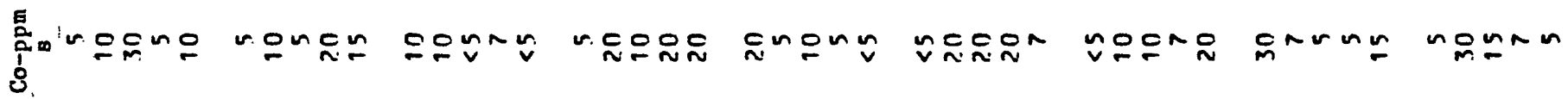

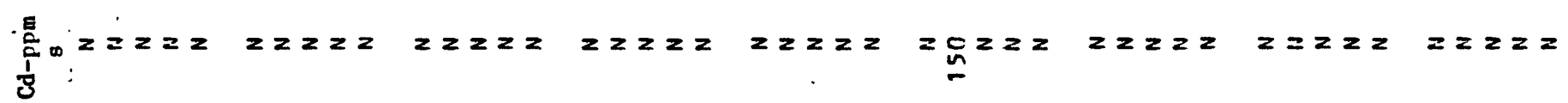

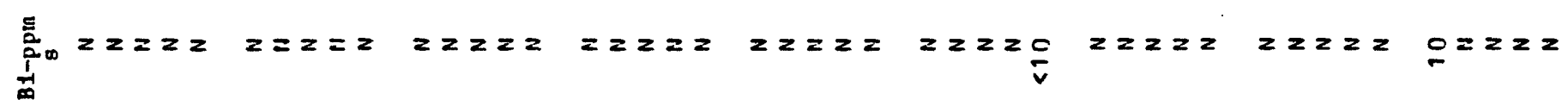

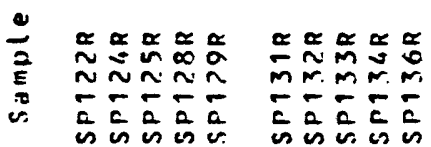

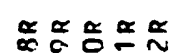
$\because \frac{0}{2}=0$ is $\frac{2}{2}=\frac{1}{2} \bar{c}$

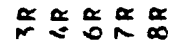

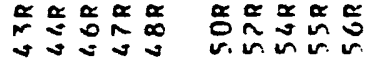

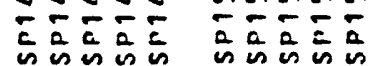

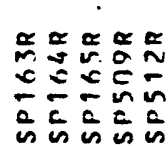

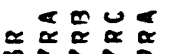
må - mmin a cis is a $\infty$

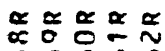

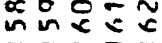
$=\bar{a}=\bar{c}$ us us us os
뜽

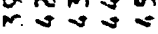
的坛的 is is is a 


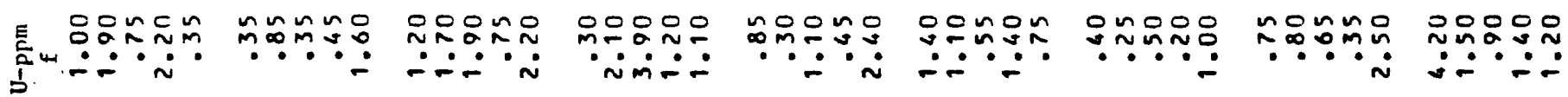

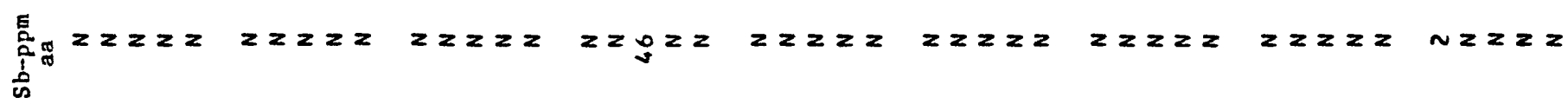

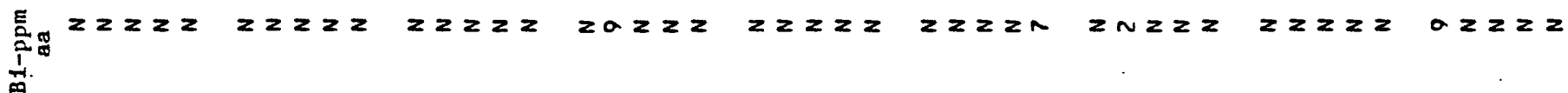

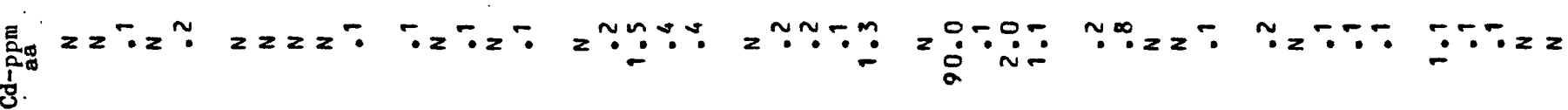

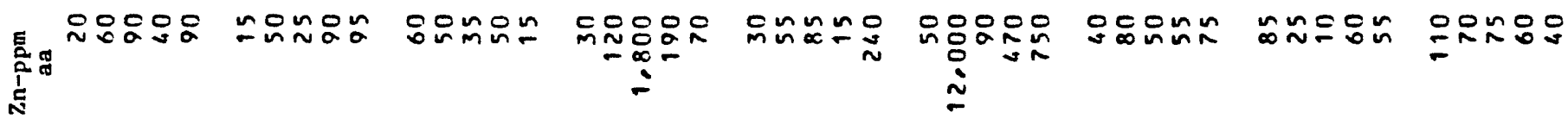

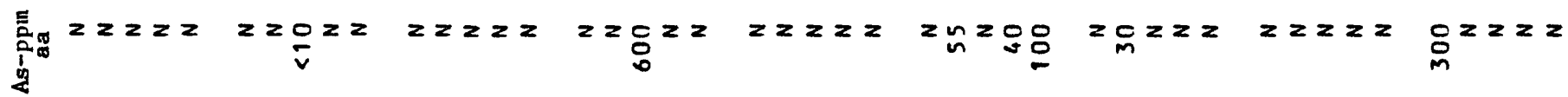

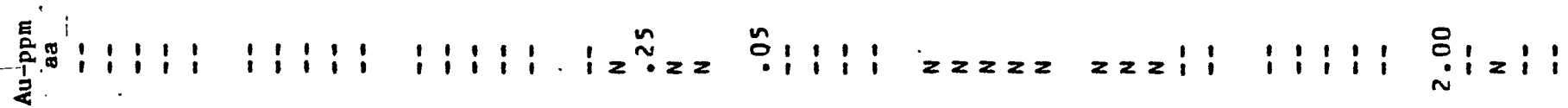

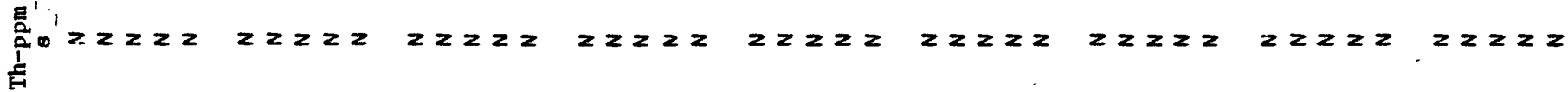
4

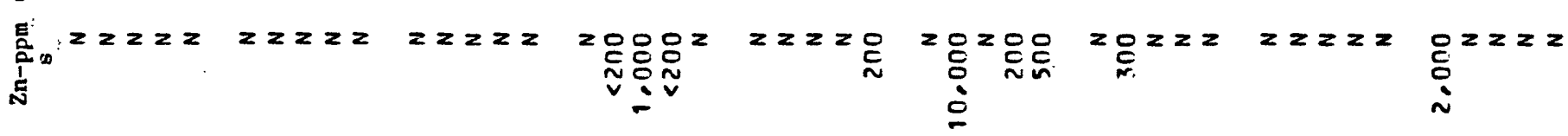
논

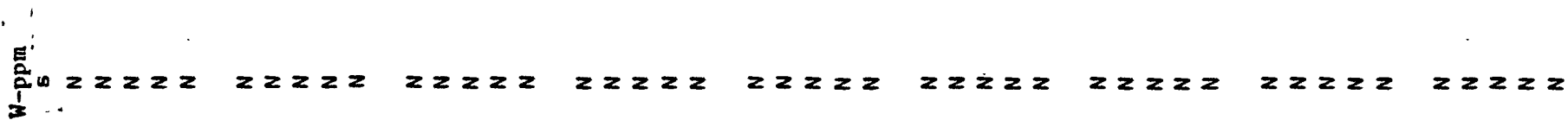

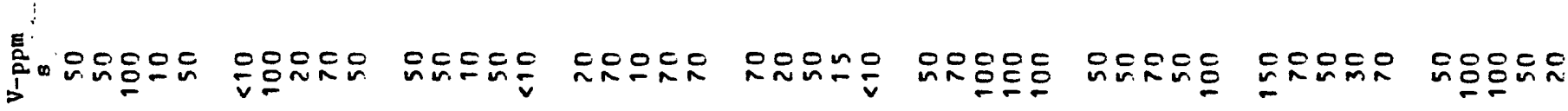

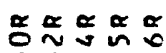

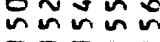
$-\frac{a}{a}-\frac{1}{a}$ un is is is in is a i es

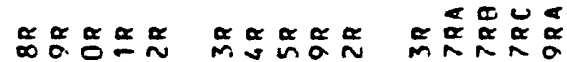

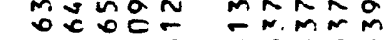
-5 - E E a a is a es ic is in a is

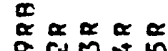
mis n in m in a i a i a i is 


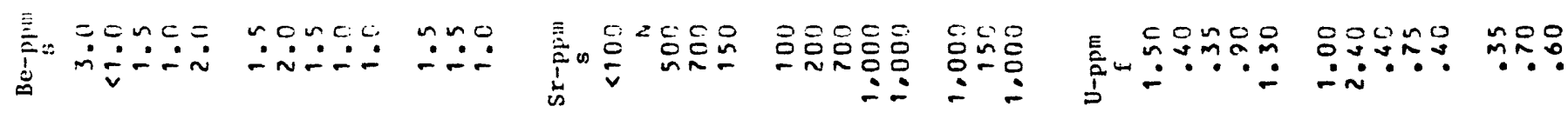

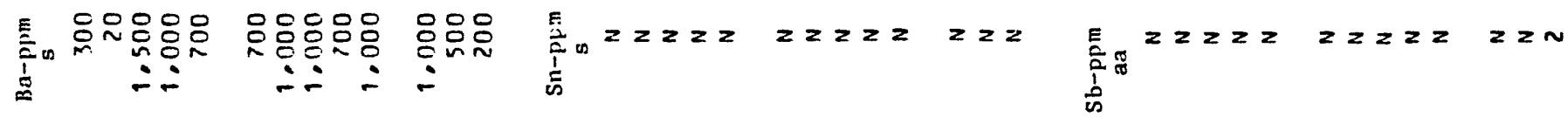

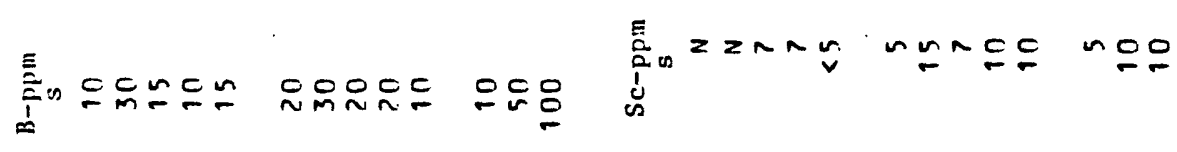

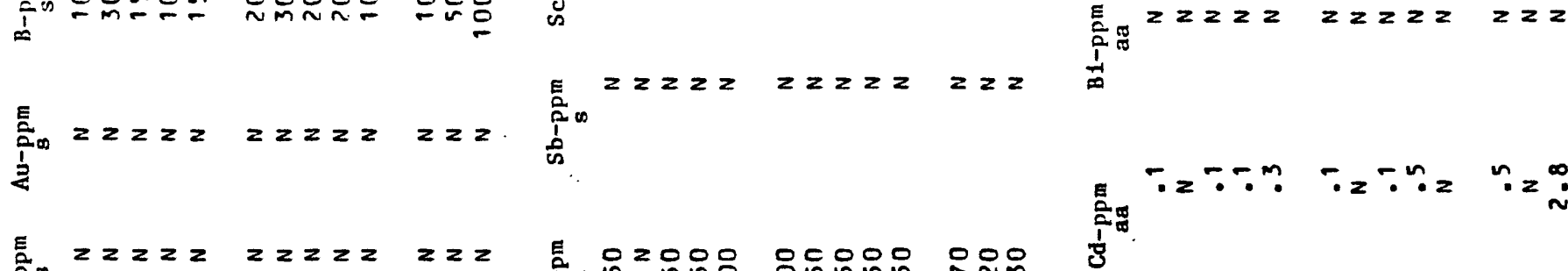

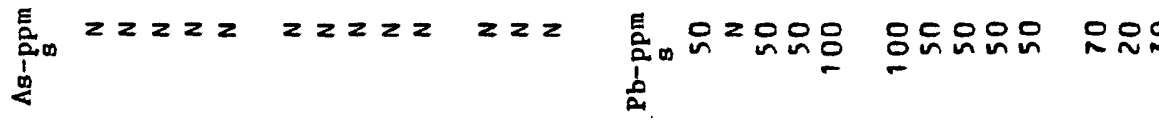

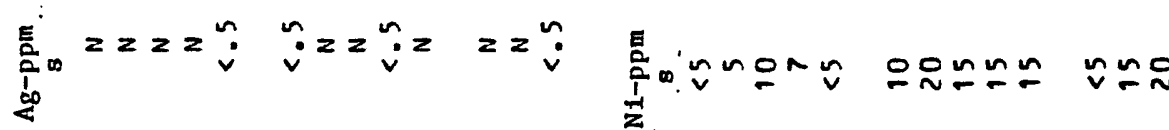

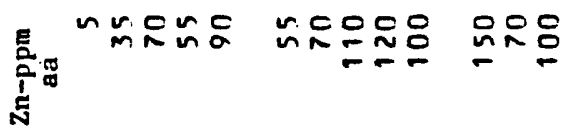

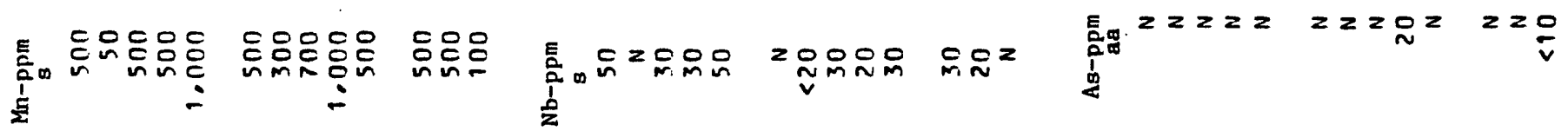

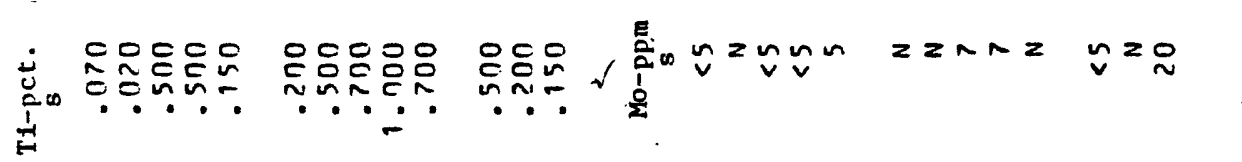

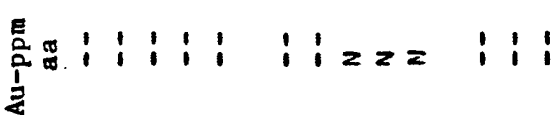

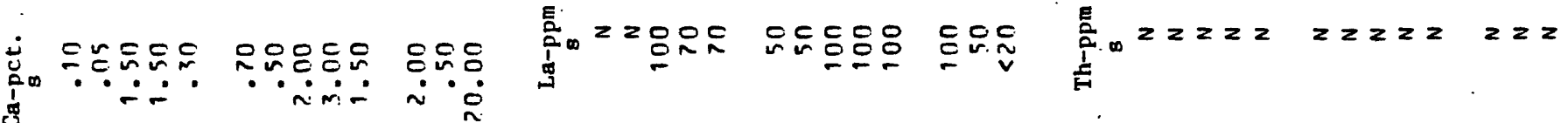

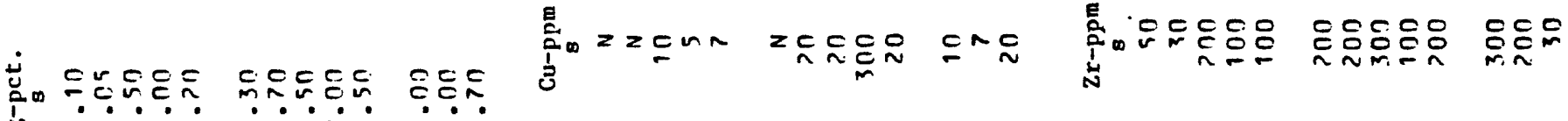

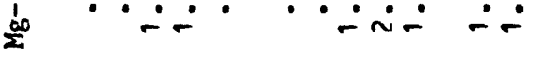

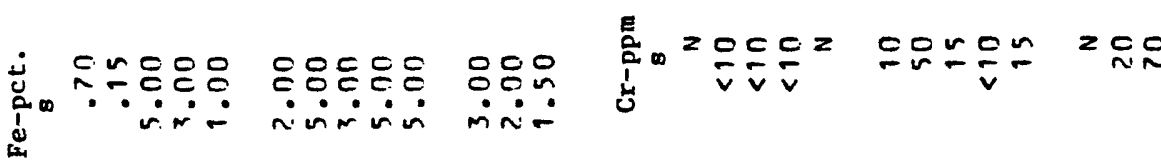

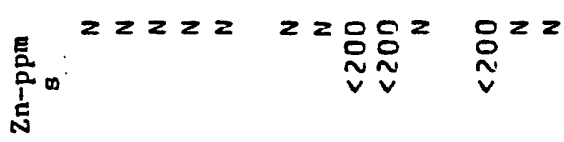

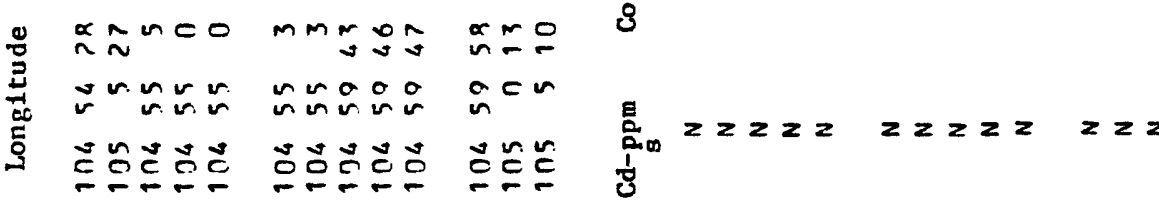

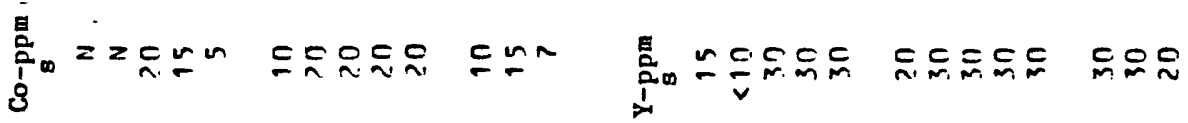
miñ

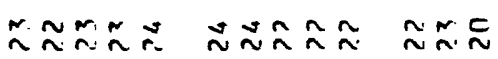

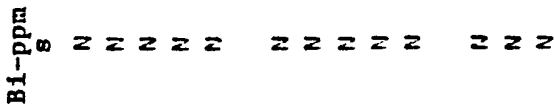

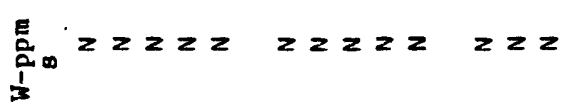

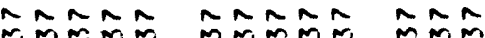

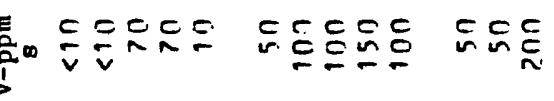




\section{Table 3.--Data for stream-sediment samples}

[Concentrations in percent for $\mathrm{Fe}, \mathrm{Mg}, \mathrm{Ca}, \mathrm{Ti}$, and in parts per million for all other elements; analyses where the column is headed by the letter $s$ were by semiquantitative emission spectrometry; analyses where the column is headed by the letters aa were by atomic absorption; and $U$ analyses were by fluorimetry; N, not detected; leaders (-), not analyzed] 


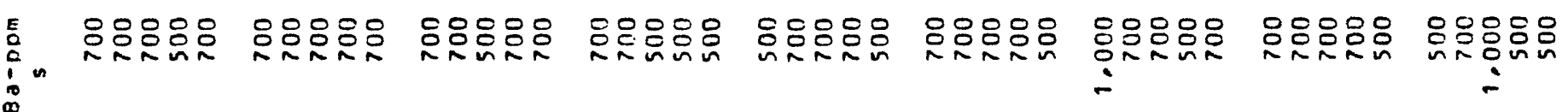

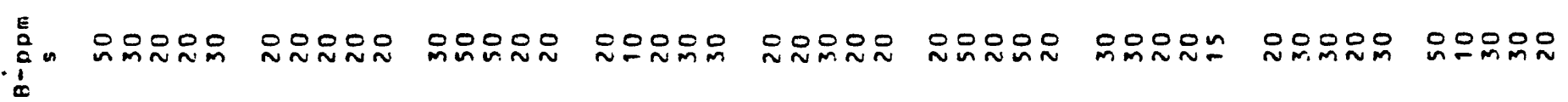
乌ุ

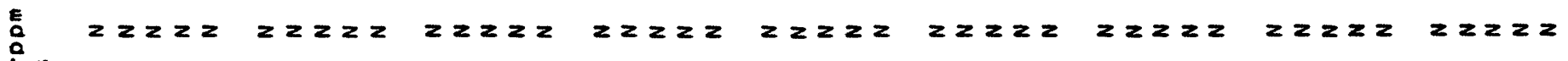

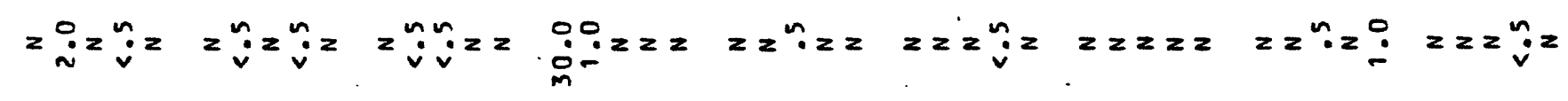

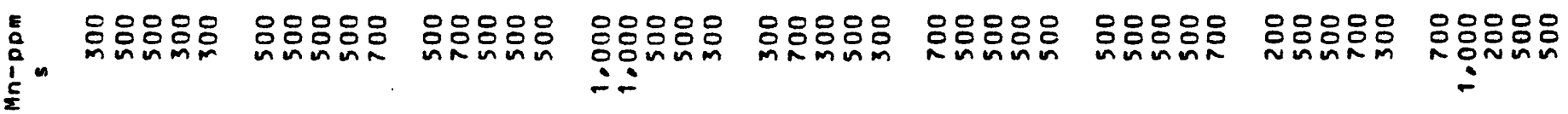

บ $\because \sim m m$ mmmn

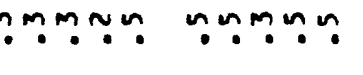

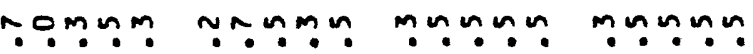

mmmッm mo? !?

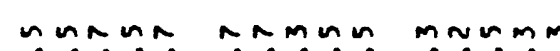

$\because \because n$

$\because ? m \backsim n$

กั?m

ค.?

$m n n m$

$\because n m m m$

๓กำ

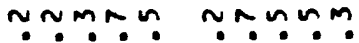

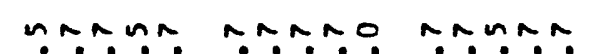

OONON NORON

$\because 0 \backsim \cap ?$

monmin

nแmon noon

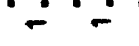

\subsection{0}

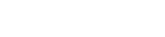

00000

00000

00000

00000

0.000

$\because 0000$

00000

00000

Nmmin miñm

mñmm

minimin sinimin

minnm

-minm

$\sim \sin$

जिं

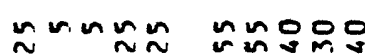

$\approx N \sim N N$

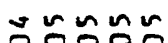

요오용

$\sim \sim \tilde{n} \backsim \tilde{n}$

苔造导导

오은

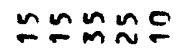

$\approx \Sigma \bar{\sim}$

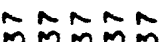

$\tilde{\sim} \sim \tilde{\sim} \dot{\sim}$

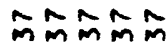

음욤요

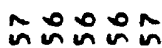

อัป์ัป

오워

$0000 \mathrm{n}$

$\operatorname{nin} \pi$

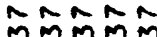

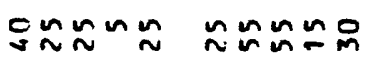

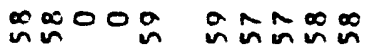

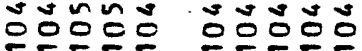

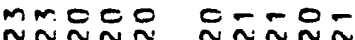

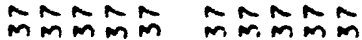

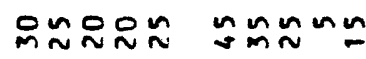
的-..

tannn 웅ㅇㅇㅇㅇ응 $m m m m m$

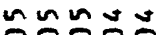

ngnmm fon m

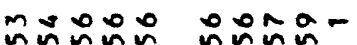

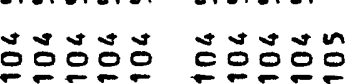

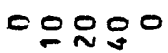
$\approx \sim \sim \sim m$ iñññ 愻的的的
D̊nッñ mmmon miñ

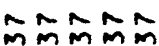

๓ュッnッ $\sim-\sim \sim \sim$ กกกN

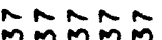

nn NNNG minninimin 
í

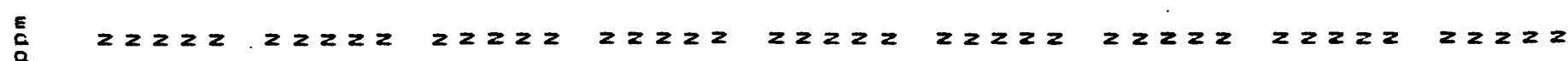
in

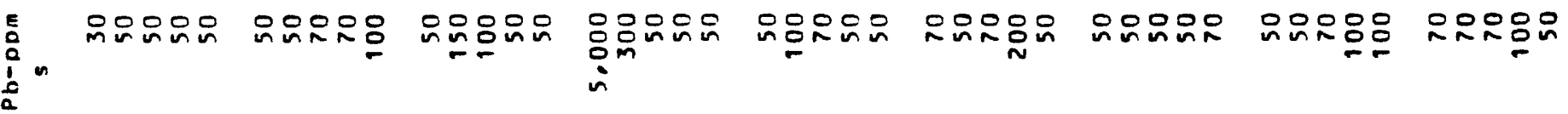

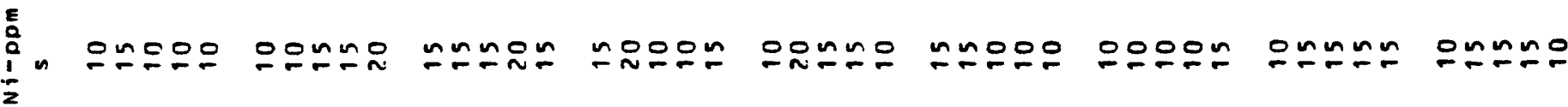
틀 윰유

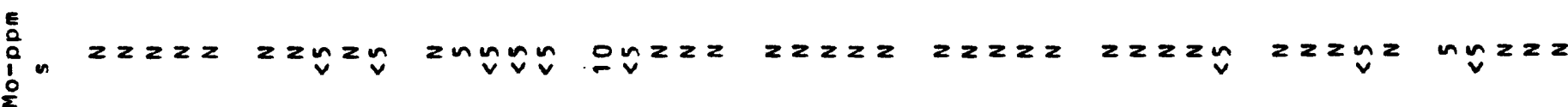

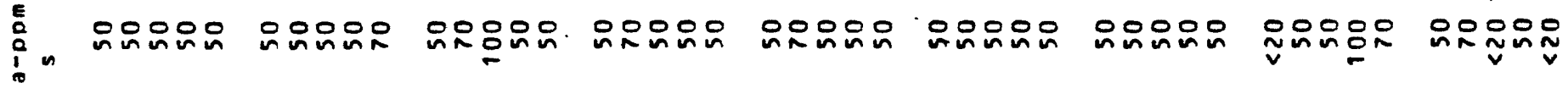
د

E 3

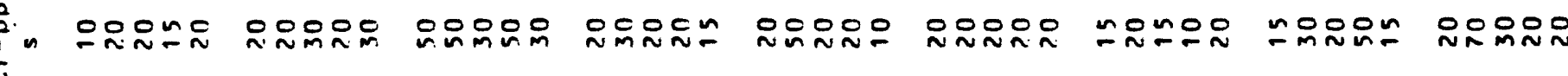

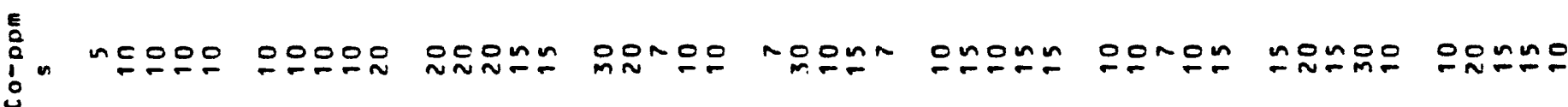

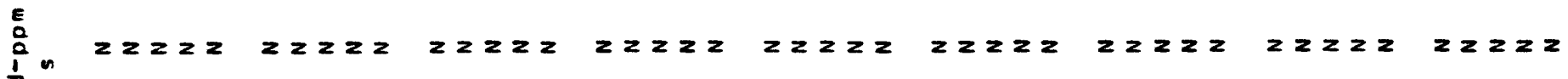
(2) 


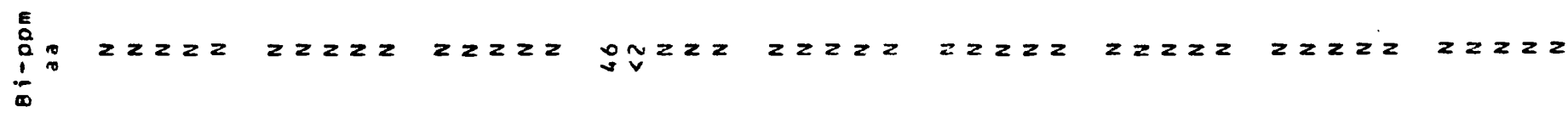
:

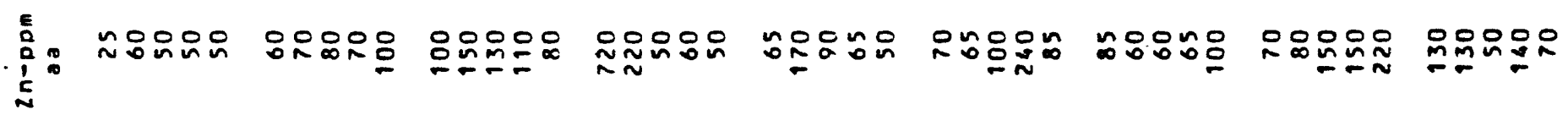

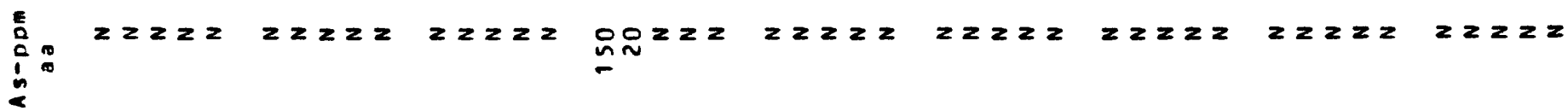

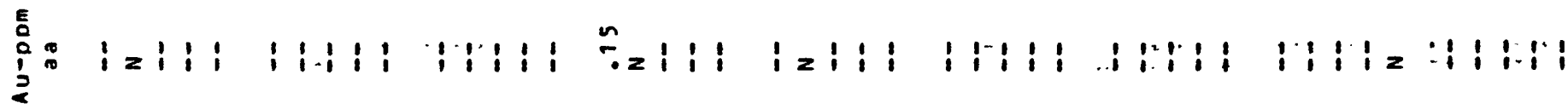

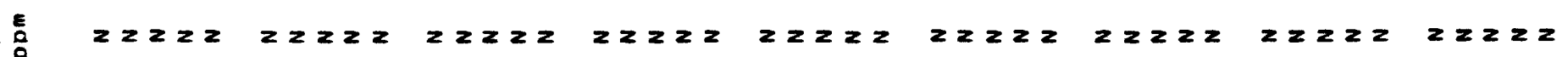

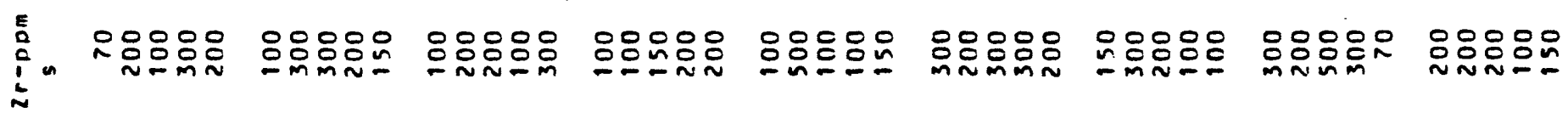

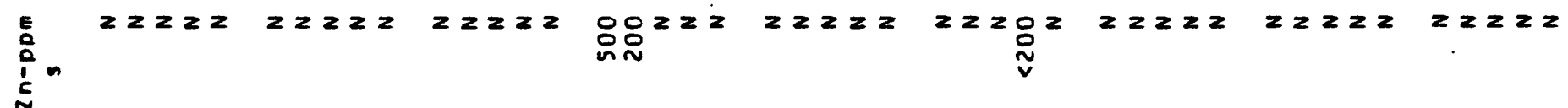

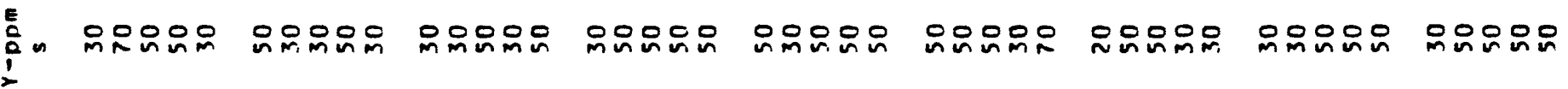

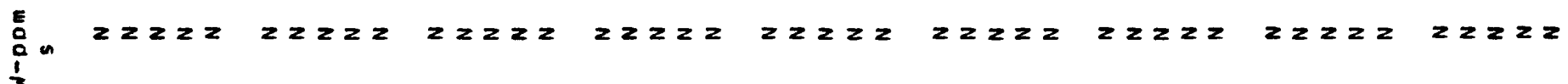

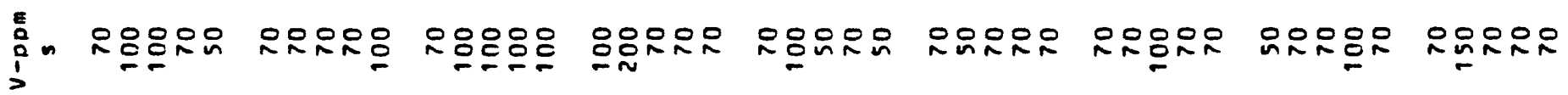

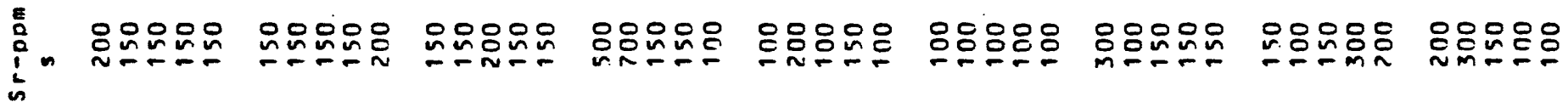
若

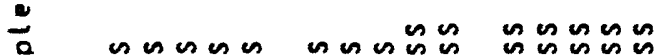

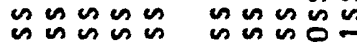
$\sim \sim \sim \sim \sim \sim \sim \infty ⿻ 上 丨$ $\sim \sim \infty \sim ⿻$

$\sin \pi n$

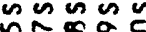
mm心an mmva 的的舟 n n n in in $\sim \sim n \sim n$ n $\therefore$ a a

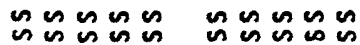

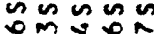

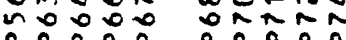
a is a a a a a a a a $\sin \sin n$ ถูก $\sim \sim \sim ⿻ \infty$ a a $a$

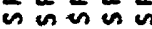

$\operatorname{nn} \sin$ in $n$ is $n$ $\sim m \sim \infty$ 0

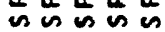




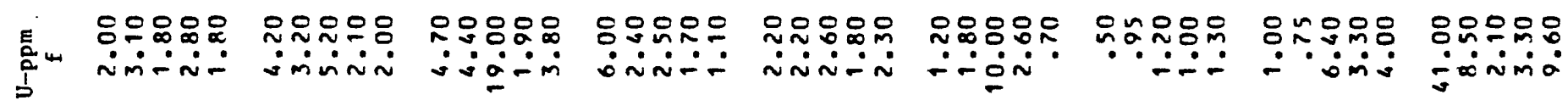

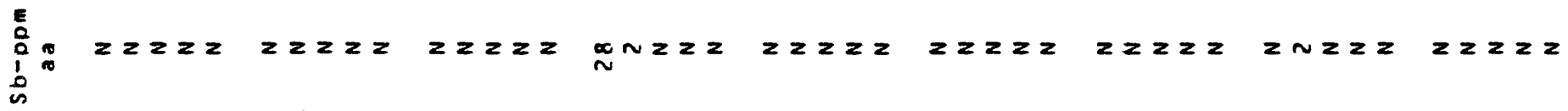

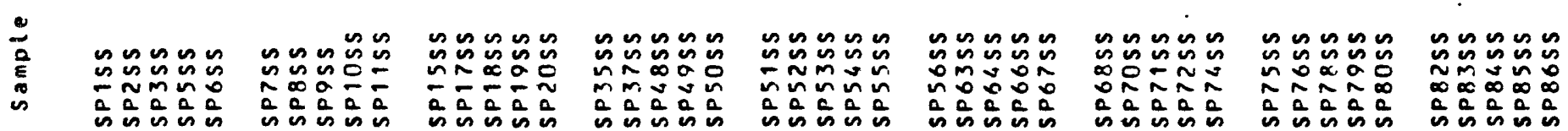




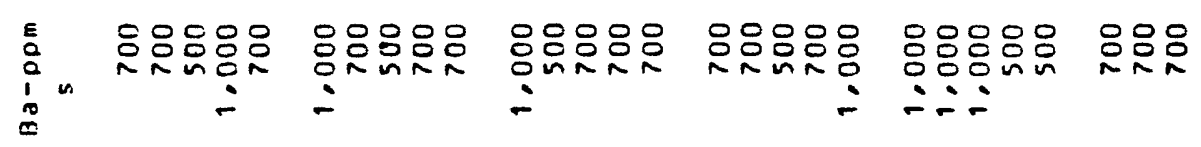

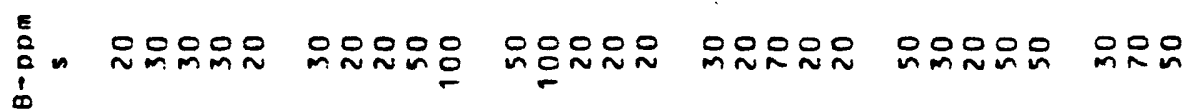

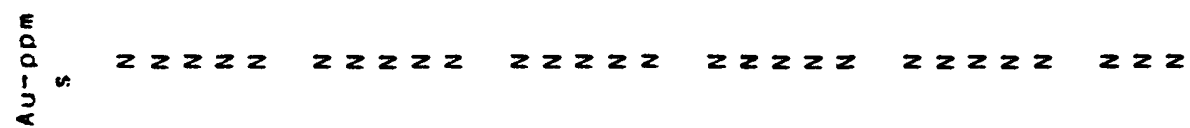

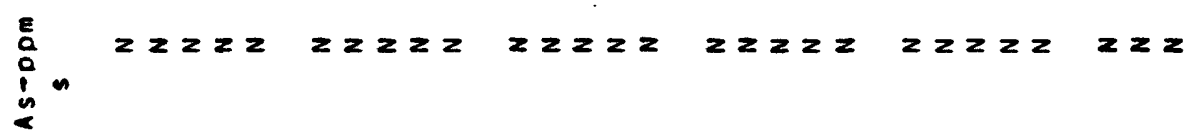

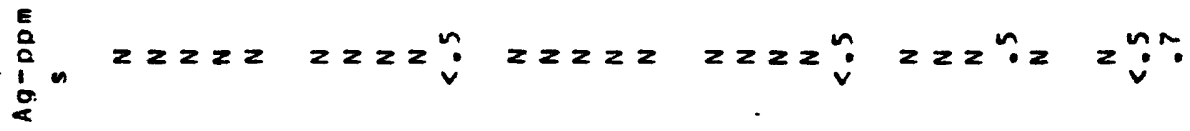

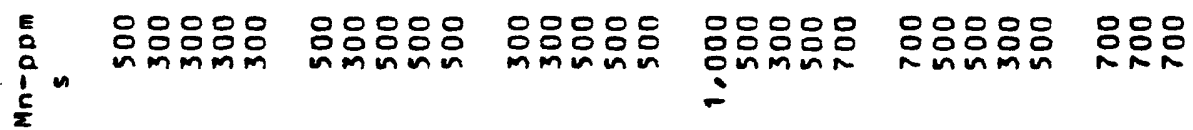
๕̆

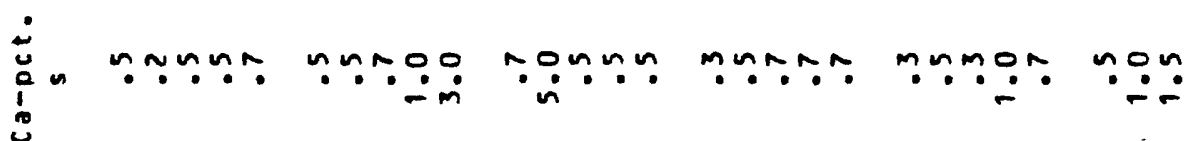

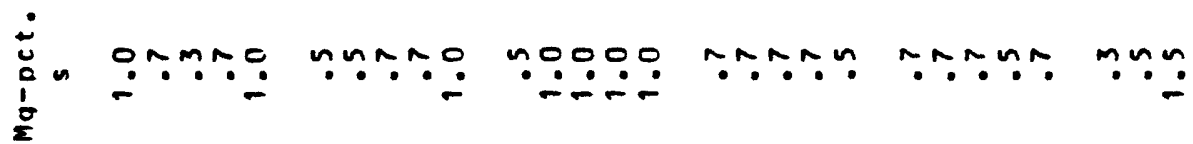
㟧

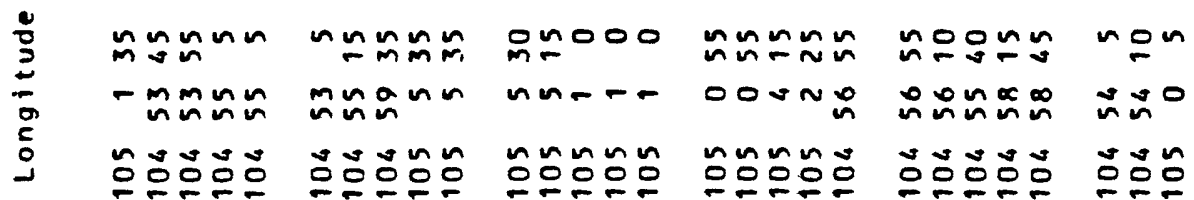

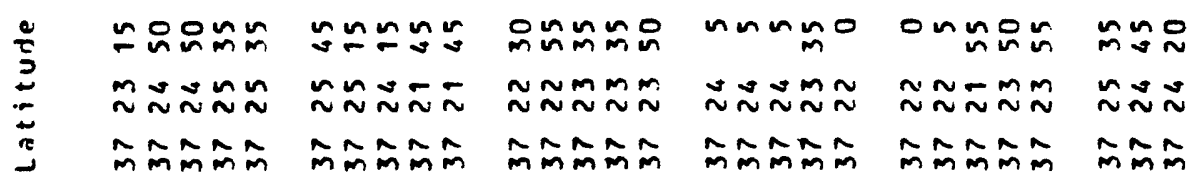




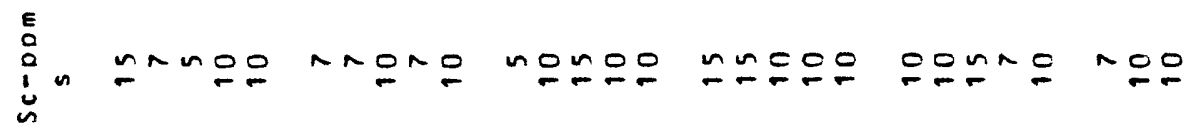

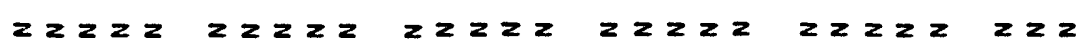

in

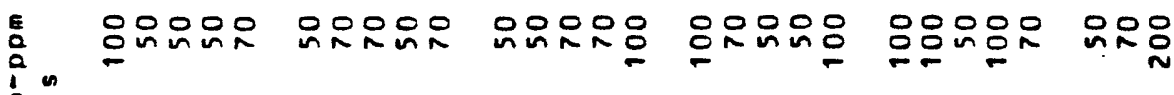

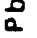

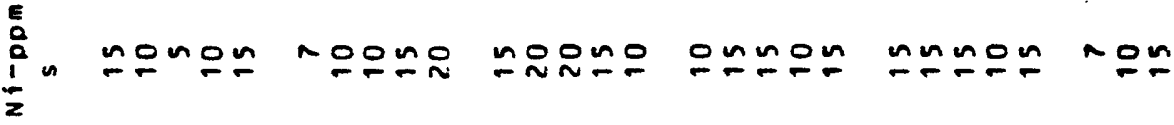

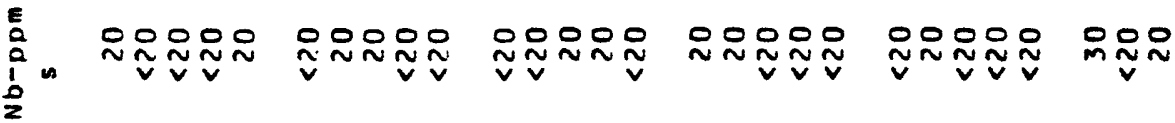

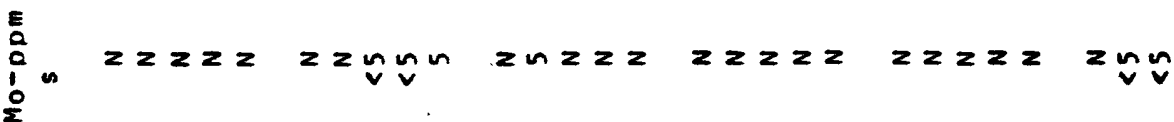

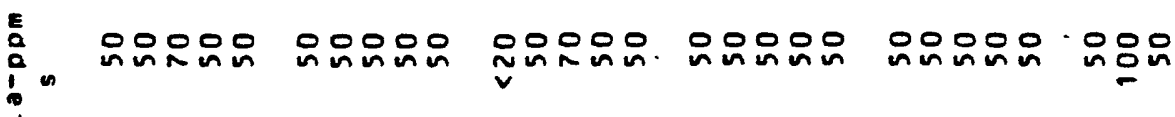

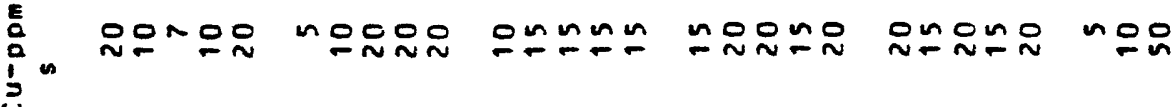

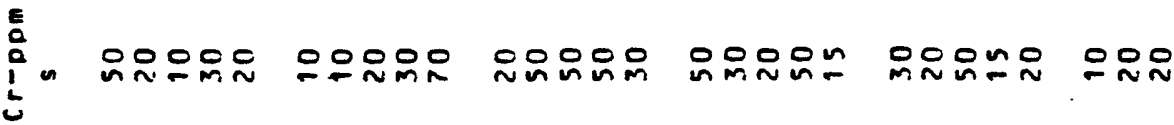

:

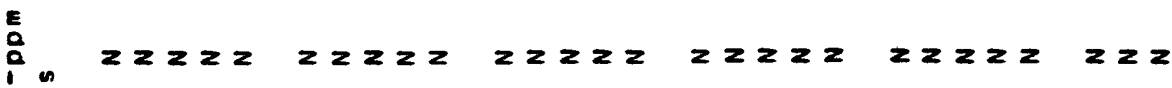

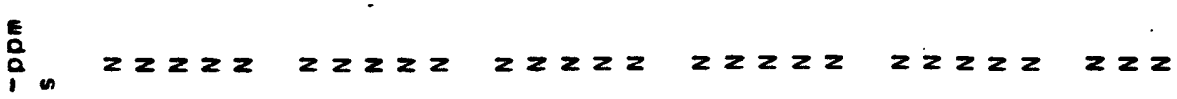

$\frac{1}{\infty}$

E

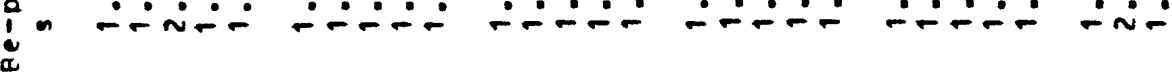

$\sin n \sin$

nis nus us

คั

융프

is in is a

nsun us $\approx n \sim n \sim$

a요 $\tilde{\sim} \tilde{n}$

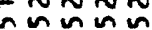

is a o a is a

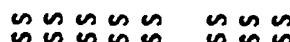

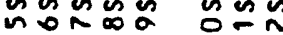

$\sim \sim \sim ⿻ 上 丨$

mnnñ munn

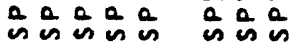




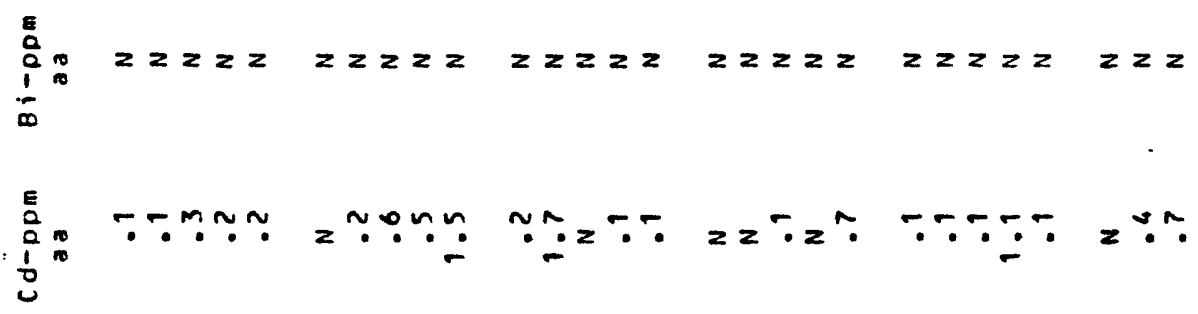

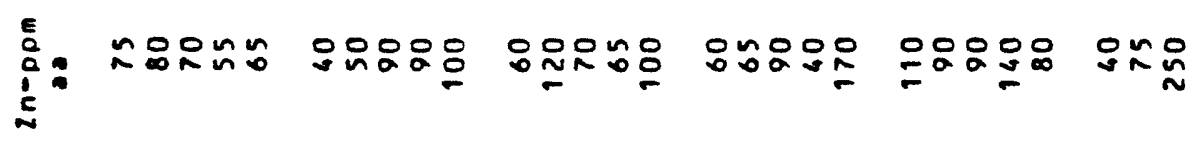

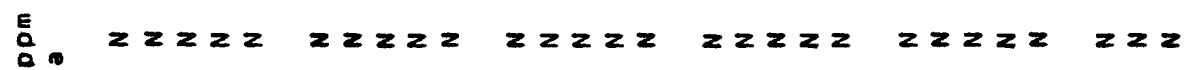
$\sum^{2}$

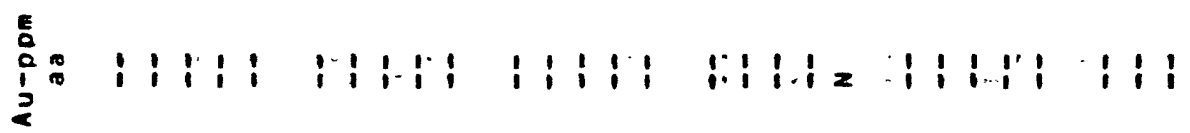

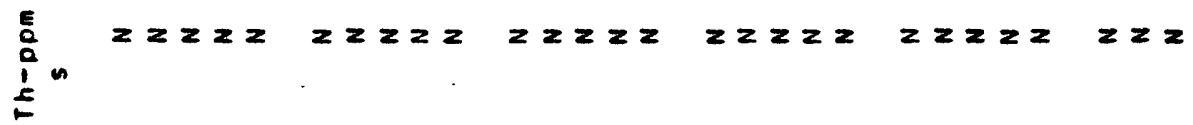

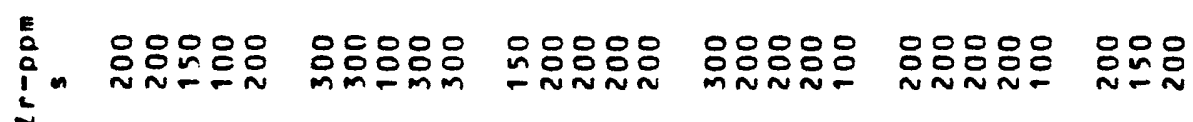

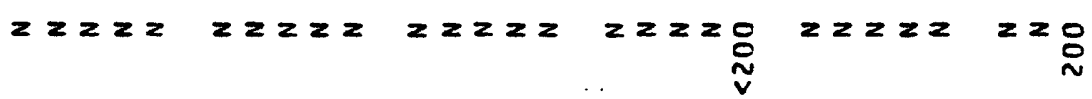

$\sum_{\substack{c \\ 0}}^{E}$

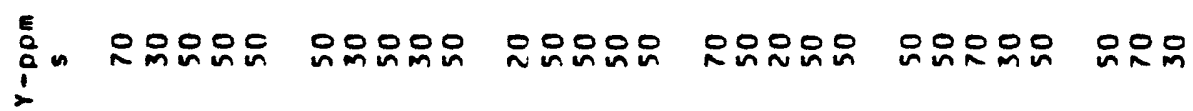
En

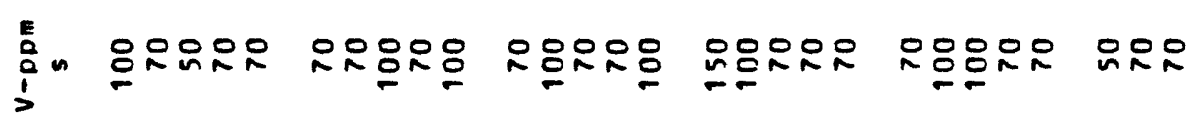

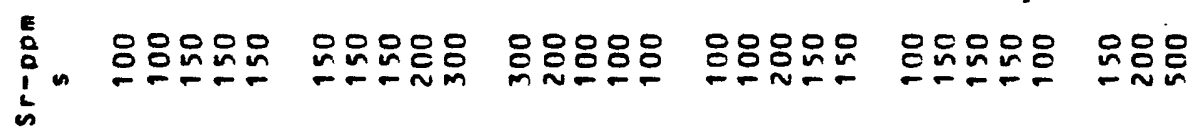

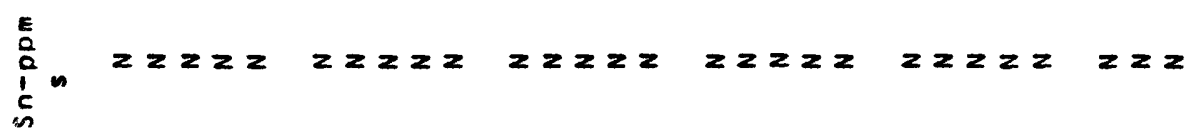

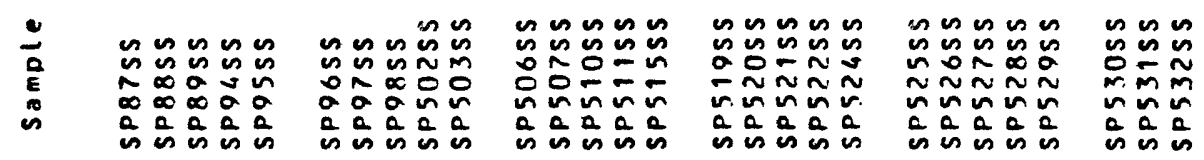




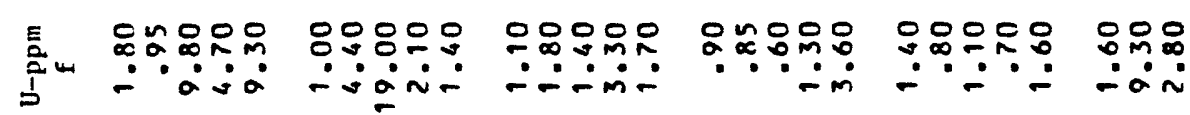

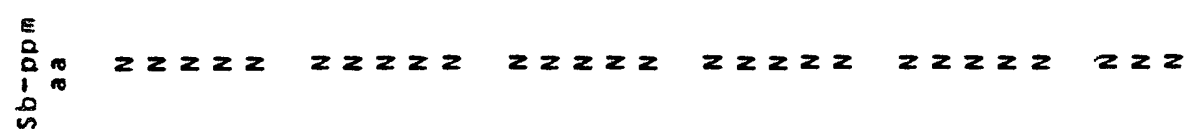


Table 4.--Data for heavy-mineral concentrate samples

[Concentrations in percent for $\mathrm{Fe}, \mathrm{Mg}, \mathrm{Ca}$, $\mathrm{Ti}$, and in parts per million for all other elements; analyses where the column is headed by the letter s were by semiquantitative emission spectrometry; N, not detected] 


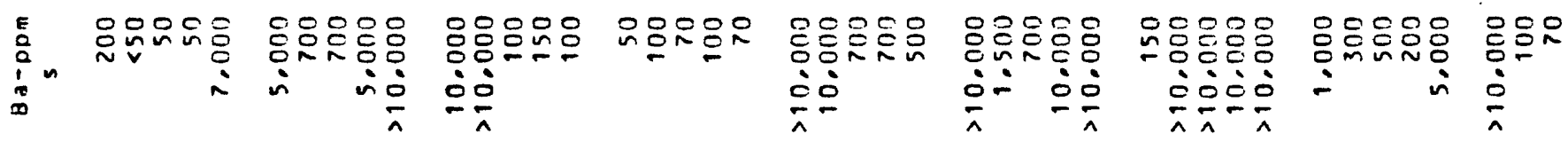

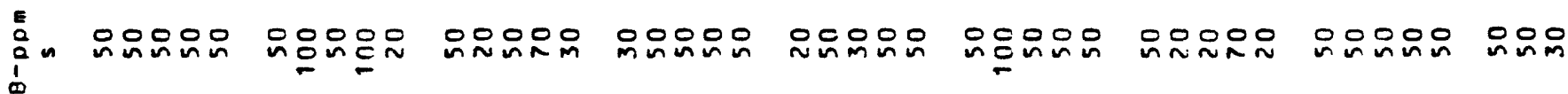

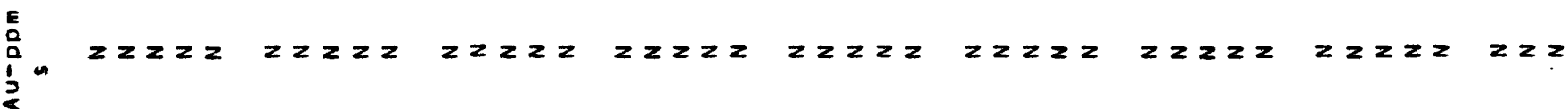
ด.

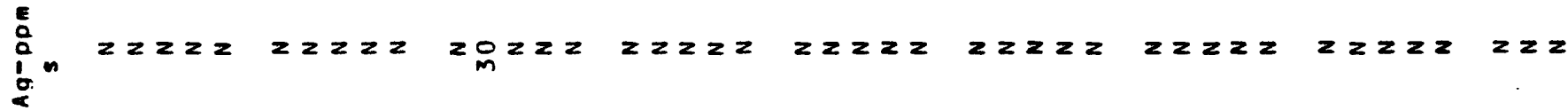

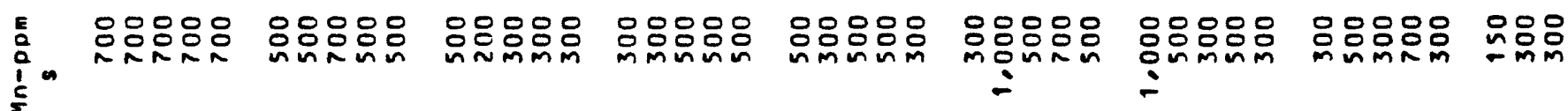

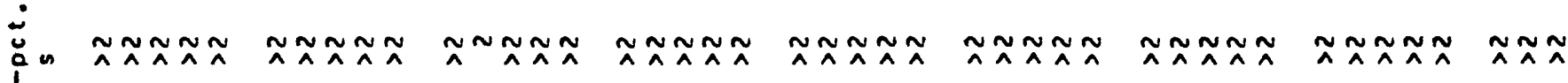
i.

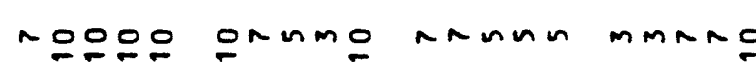
s

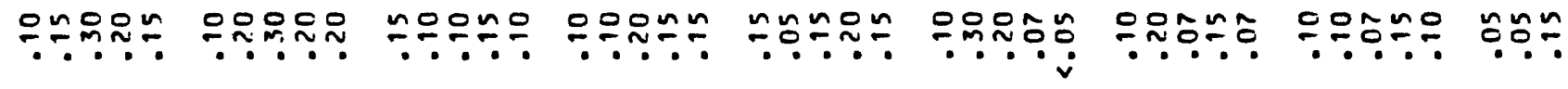

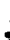

$\operatorname{non} \operatorname{sic}$

onn no

ronmo

no:ma

oon

$0000 \mathrm{~m}$

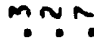<smiles>C1CCCCC1</smiles>

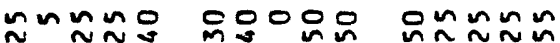
$\sim \sim \sim \sim n$

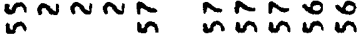
nin $\operatorname{mon}$

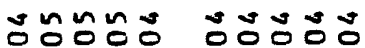

\section{soños} 웅ㅇㅇㅇㅇㅇ

으능요 $\infty_{n}^{\infty}--$

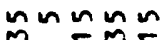
mmm我 ปี่ณnณ $\ln 2020$ 응영ㅇㅇㅇ 응으유 유융ㅇㅇ

\section{으ำnnm} nco0o กับับ $\sin a a^{2}$ ตn 幺幺ำ in 0000 $n n \backsim \backsim n$ mon n NONNN ำกก̃ $n \tilde{M} N \tilde{m} N N N N N$

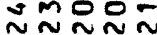
$\tilde{M} \tilde{m} \tilde{m} \tilde{m}$

$\hat{m} \tilde{m} N \hat{m}$ $n N N N N$ m̃nmm

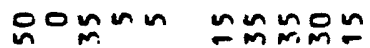
ก̃-ñ nnmun $\sin 20$ 으웅응응 onnmn 엉ㅇㅁ응으

음ำm ปักละn $\sim \sim \sim N N$ mMnmm̃ numon $\tilde{\sim} \check{\sim} \tilde{N}$ MñmMn oo $\approx \sim \sim$ - - On:

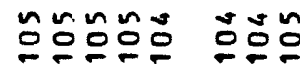

mmnno Oñ

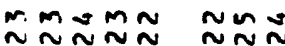

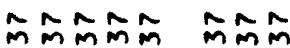




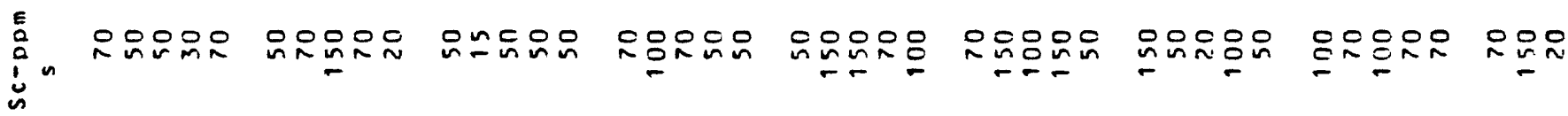

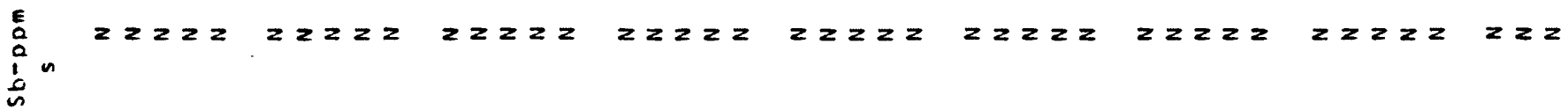
เั

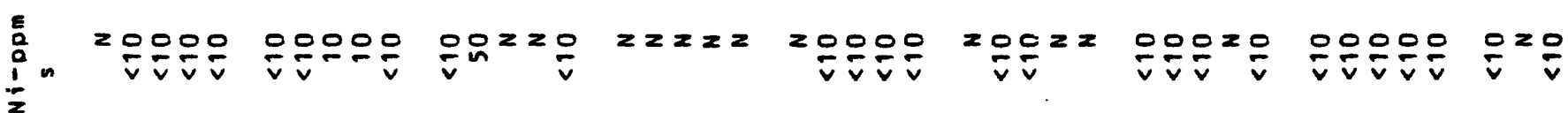

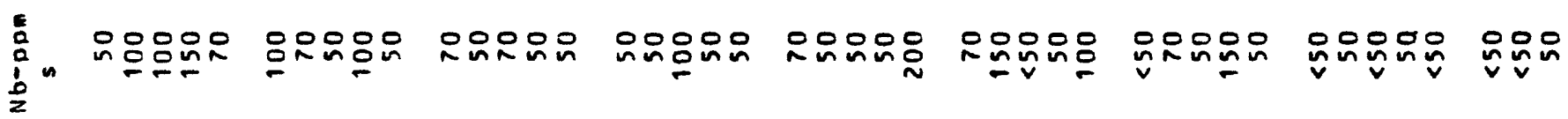

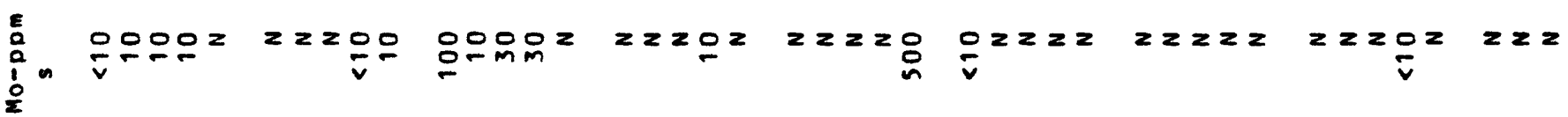
ปั

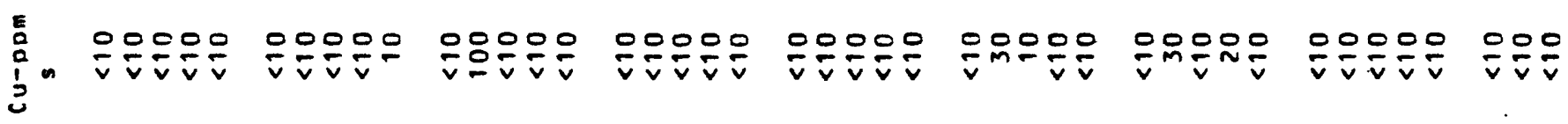
ปร "ִ

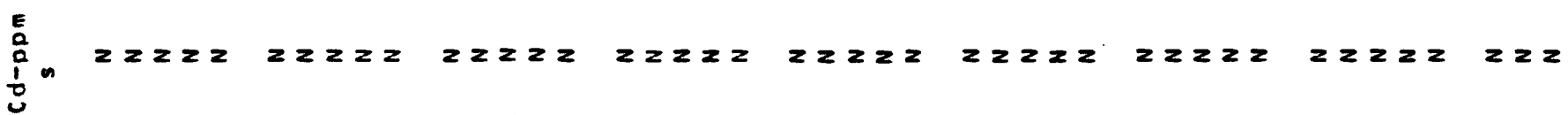
ป. 1.

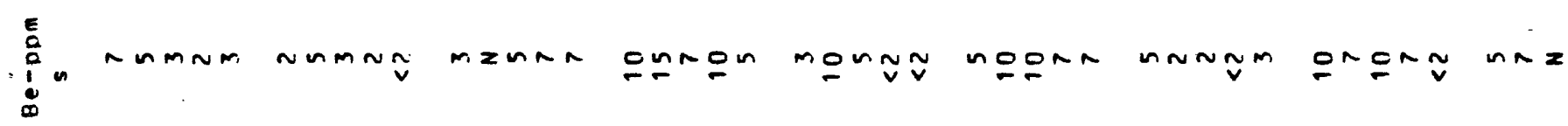

บ范范范

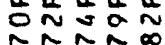
is is is is

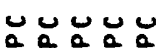

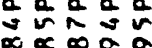
$\infty \propto \infty a$ is a $\frac{a}{n}$ is
ن芒范

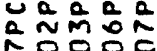
aㅇํ의 a ต $\therefore \frac{a}{n} \frac{a}{n} \frac{a}{n}$

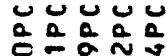
ñom

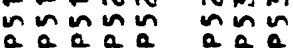
is is a i i is is 


\section{응요음}

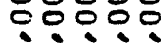

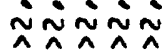

응응응

웅요

$\dot{\sim} \sim \dot{\wedge} \dot{\wedge}$

응융응

00000

inini

응응융

$\because \because: ?:$

응등융

$\because 000$

응응융

00000

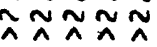

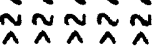

응용응융

00000

$\dot{\sim} \sim \dot{\sim} \sim$

용용요 용응

움드

iñin $\sim \dot{\sim} \sim$

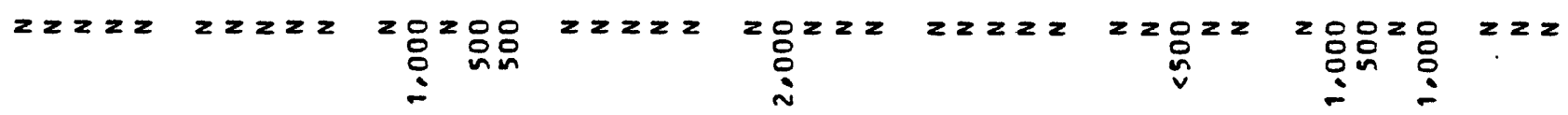

응움용융요

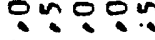

음윰융요

옹응응

응유뭈요

응응용용요

등ㅇㅇㅇㅇㅇㅇ응

는ㅁํํํำ

양융융

옹음용윰윰

응응응용 응윰

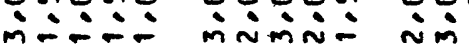

E

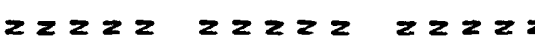

$22222 \quad 2222 z$

$z z z z z$

$z z z z z$

$z z z z z \quad z z z$

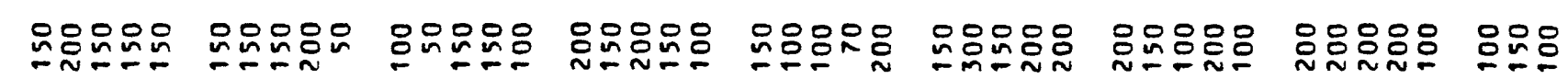

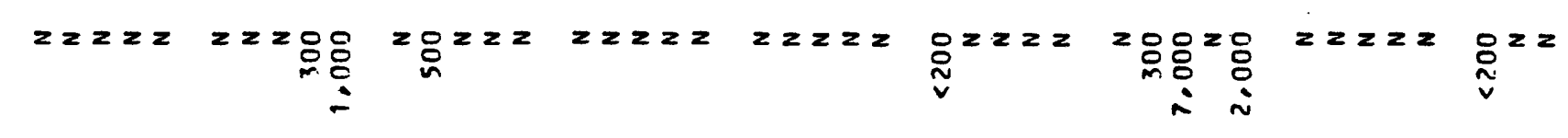

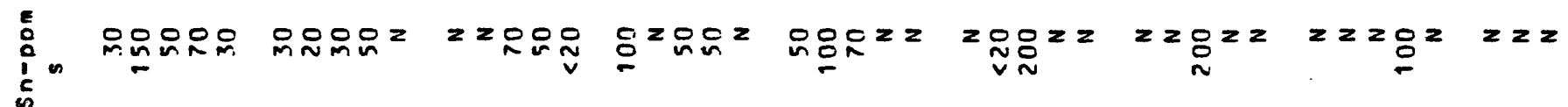




\section{REFERENCES}

Budding, K. E., and Kluender, S. E., 1983, Mineral resource potential map of the Spanish Peaks Wilderness Study Area, Huerfano and Las Animas Counties, Colorado: U.S. Geological Survey Miscellaneous Field Studies Map MF-1542-C, scale 1:50,000.

Budding, K. E., and Lawrence, V. A., 1983a, Geochemical maps of the Spanish Peaks Wilderness Study Area, Huerfano and Las Animas Counties, Colorado: U.S. Geological Survey Miscellaneous Field Studies Map MF-1542-B, scale $1: 50,000$.

1983b, Reconnaissance geologic map of the Spanish Peaks Wilderness Study Area, Huerfano and Las Animas Counties, Colorado: U.S. Geological Survey Miscellaneous Field Studies Map MF-1542-A, scale 1:50,000. 